ANL/NSE-21/23

INL/EXT-21-63185

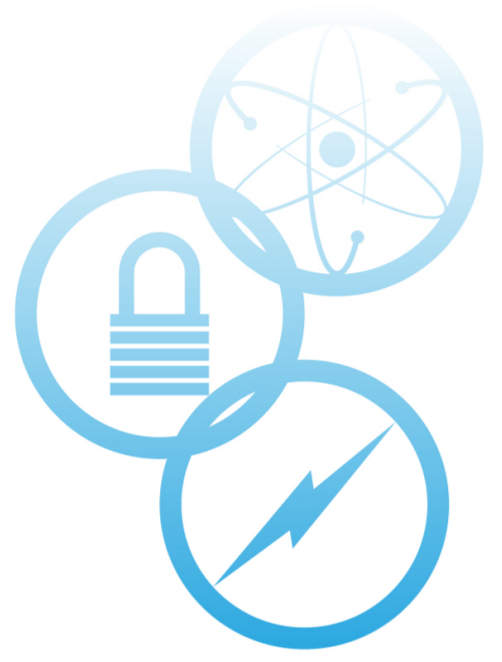

\title{
Griffin Software Development Plan
}

Changho Lee ${ }^{1}$, Yeon Sang Jung ${ }^{1}$, Hansol Park ${ }^{1}$, Emily R. Shemon ${ }^{1}$ and Javier Ortensi ${ }^{2}$, Yaqi Wang ${ }^{2}$, Vincent M. Labouré ${ }^{2}$, Zachary Prince ${ }^{2}$

${ }^{1}$ Nuclear Science and Engineering Division, Argonne National Laboratory

${ }^{2}$ Nuclear Science and Technology Division, Idaho National Laboratory
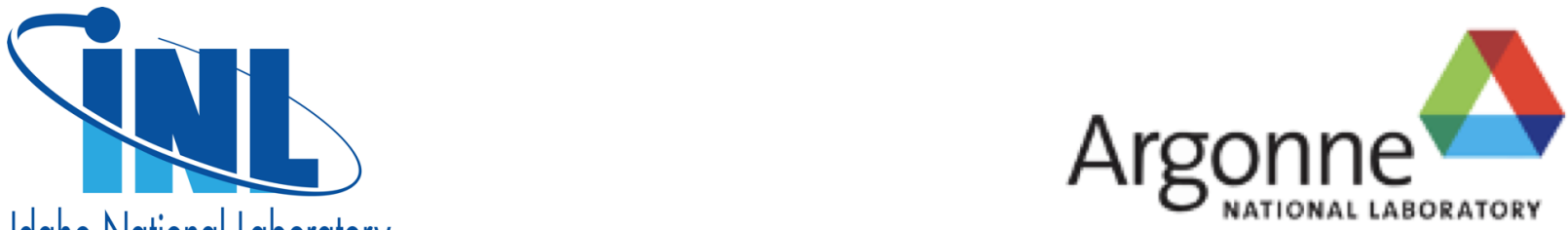

Idaho National Laboratory 


\section{DISCLAIMER}

This information was prepared as an account of work sponsored by an agency of the U.S. Government. Neither the U.S. Government nor any agency thereof, nor any of their employees, makes any warranty, expressed or implied, or assumes any legal liability or responsibility for the accuracy, completeness, or usefulness, of any information, apparatus, product, or process disclosed, or represents that its use would not infringe privately owned rights. References herein to any specific commercial product, process, or service by trade name, trade mark, manufacturer, or otherwise, does not necessarily constitute or imply its endorsement, recommendation, or favoring by the U.S. Government or any agency thereof. The views and opinions of authors expressed herein do not necessarily state or reflect those of the U.S. Government or any agency thereof. 
ANL/NSE-21/23

INL/EXT-21-63185

\section{Griffin Software Development Plan}

Changho Lee ${ }^{1}$, Yeon Sang Jung ${ }^{1}$, Hansol Park ${ }^{1}$, Emily R. Shemon ${ }^{1}$ and Javier Ortensi ${ }^{2}$, Yaqi Wang ${ }^{2}$, Vincent M. Labouré ${ }^{2}$, Zachary Prince ${ }^{2}$

${ }^{1}$ Nuclear Science and Engineering Division, Argonne National Laboratory

${ }^{2}$ Nuclear Science and Technology Division, Idaho National Laboratory

June 2021

\section{Argonne National Laboratory Idaho National Laboratory}

Prepared for the U.S. Department of Energy Office of Nuclear Energy Under UChicago Argonne, LLC Contract DE-AC02-06CH11357 Under DOE Idaho Operations Office Contract DE-AC07-05ID14517 
Page intentionally left blank 


\section{SUMMARY}

Griffin is a MOOSE-based reactor physics application for advanced reactor multiphysics modeling and simulation. The application is developed in a consistent multiphysics environment with strong software quality assurance. Griffin inherited most of the capabilities of MAMMOTH/Rattlesnake and is adopting the capabilities from PROTEUS that are needed in the code. The toolset includes a variety of deterministic radiation transport solvers for fixed source, k-eigenvalue, adjoint, and subcritical multiplication, as well as transient solvers for point-kinetics, improved quasistatic, and spatial dynamics. The code contains the cross-section preparation capabilities applicable to fast and thermal reactors, including TRISO-fueled reactors. Core management capabilities include core performance, fuel depletion and shuffling, equilibrium core calculation, pebble-bed reactor run-in and equilibrium core, molten-salt reactor delayed neutron precursor drift, and control rod and drum movement with cusping correction. This software development plan presents the current and future capabilities and features in Griffin for the design and analysis of non-lightwater reactor systems in steady-state and transient conditions. 


\section{ACKNOWLEDGEMENTS}

This work was supported by the Nuclear Energy Advanced Modeling and Simulation Program managed the U.S. Department of Energy, Office of Nuclear Energy. Accordingly, the U.S. Government retains a nonexclusive, royalty-free license to publish or reproduce the published form of this contribution, or allow others to do so, for U.S. Government purposes. 
Page intentionally left blank 


\section{CONTENTS}

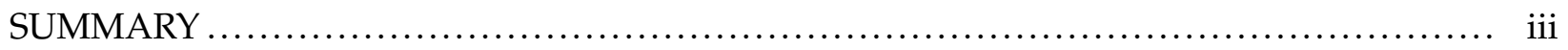

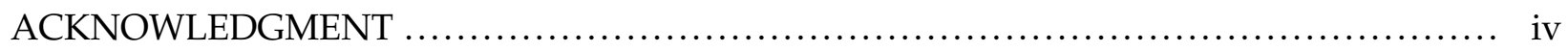

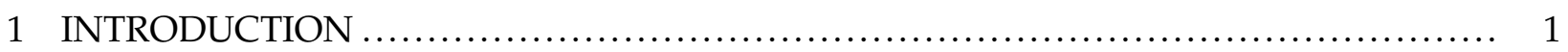

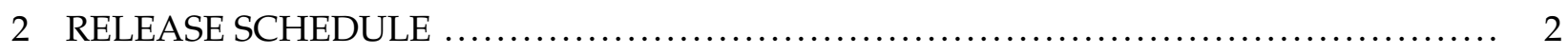

$3 \quad$ FUNCTIONAL REQUIREMENTS FOR REACTOR PHYSICS $\ldots \ldots \ldots \ldots \ldots \ldots \ldots \ldots \ldots \ldots .2$

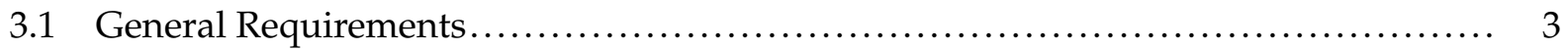

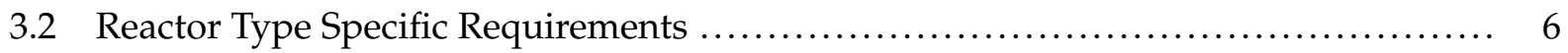

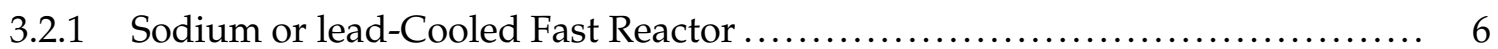

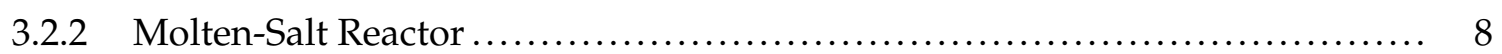

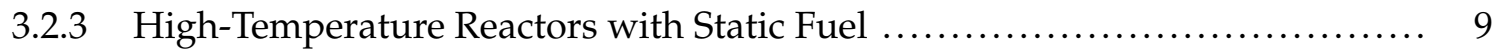

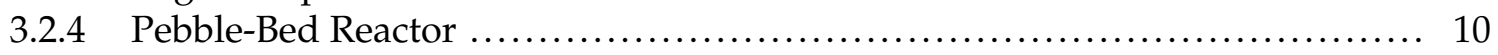

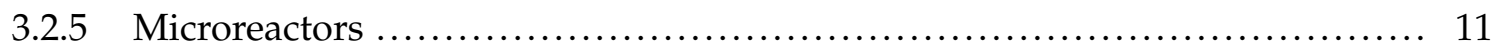

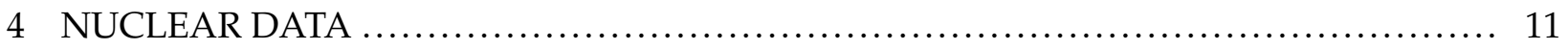

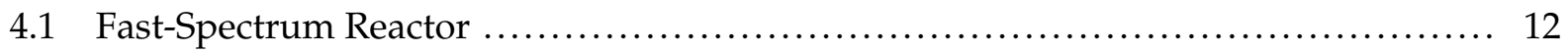

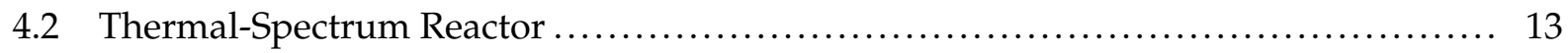

4.3 Equivalence Parameters. ................................................. 15

4.4 Representation of Cross Sections and Equivalence Data. $\ldots \ldots \ldots \ldots \ldots \ldots \ldots \ldots \ldots \ldots$

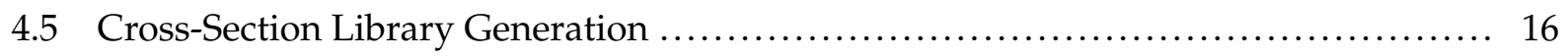

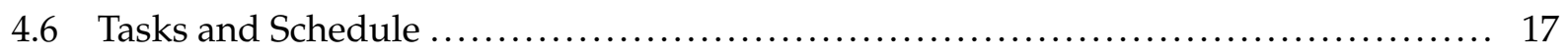

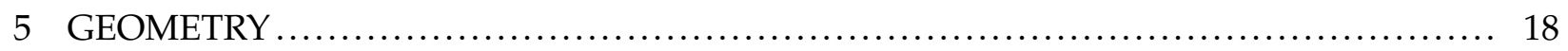

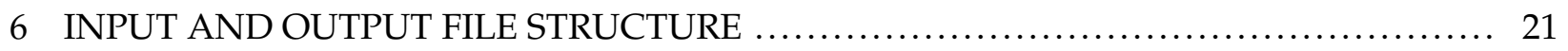



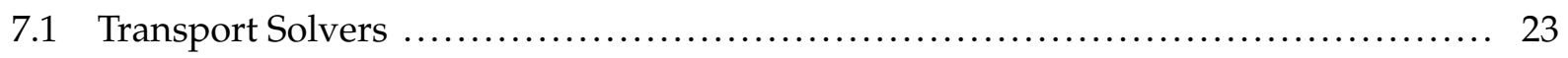

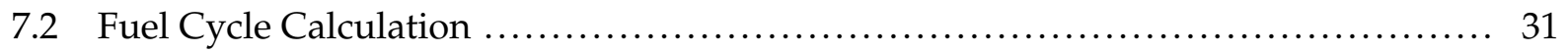

7.3 Perturbation, Sensitivity and Uncertainty Quantification $\ldots \ldots \ldots \ldots \ldots \ldots \ldots \ldots \ldots . \ldots 2$

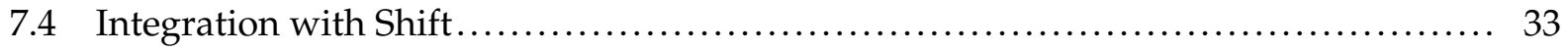




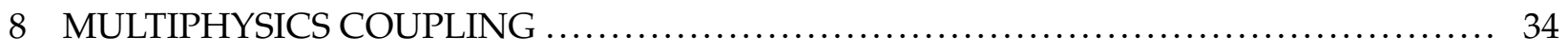



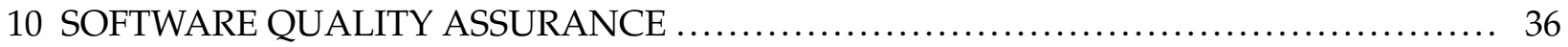

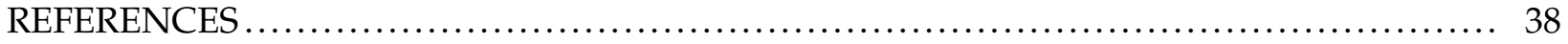




\section{TABLES}

Table A1 Summary of the Capabilities and Features of Griffin - Part 1. .................... 45

Table A2 Summary of the Capabilities and Features of Griffin - Part 2. . ................... 46

Table A3 Summary of the Capabilities and Features of Griffin - Part 3. ..................... 47

Table A4 Summary of the Capabilities and Features of Griffin - Part 4....................... 49 


\section{ACRONYMS}

API application programming interface

BG broad group

CMFD coarse-mesh finite difference

CRAM Chebyshev rational approximation method

CSAPI cross section application programming interface

DCC depressurised conduction cooldown

DLOFC depressurised loss of forced cooling

DPA displacements per atom

DSA diffusion synthetic acceleration

FHR fluoride high-temperature reactor

HTR high-temperature reactor

LDRD laboratory directed research and development

LFR Lead-cooled fast reactor

MMPA minimax polynomial approximation

MOOSE multiphysics object-oriented simulation environment

MSR molten-salt reactor

NDA nonlinear diffusion acceleration

PBR pebble-bed reactor

PCC pressurised conduction cooldown

PKE point kinetics equation

PLOFC pressurised loss of forced cooling

PN spherical harmonics

S2TA S2 transport acceleration

SFR sodium-cooled fast reactor

SN discrete ordinate

TRISO Tristructural Isotropic

UFG ultrafine group 
ULOF unprotected loss of flow

ULOHS unprotected loss of heat sink

UTOP unprotected transient overpower 
Page intentionally left blank 


\section{INTRODUCTION}

The Nuclear Energy Advanced Modeling and Simulation (NEAMS) campaign of the Reactor Fleet and Advanced Reactor Development Office, with the U.S. Department of Energy's Office of Nuclear Energy (DOE-NE), has developed a number of advanced modeling and simulation (M\&S) tools to accelerate the deployment of advanced nuclear energy technologies.

Until FY 2020, the MC²-3 [1]/PROTEUS [2] toolset has been developed on the SHARP multiphysics framework [3] under the NEAMS program at Argonne National Laboratory (ANL). Also, Idaho National Laboratory (INL) has developed the Rattlesnake radiation transport solver [4] and the MAMMOTH reactor multiphysics application [5] using the MOOSE multiphysics framework [6] under laboratory directed research and development (LDRD) projects and later NEAMS. In early FY 2020, the two laboratories agreed on the joint development of an advanced reactor physics tool named Griffin using the multiphysics object-oriented simulation environment (MOOSE) framework, based on the integration of the two code suites (MAMMOTH/Rattlesnake and $\mathrm{MC}^{2}-3 /$ PROTEUS). This development strategy is intended to focus NEAMS reactor physics efforts on the development of a single code to effectively meet the needs of users, including industry and government organizations in a timely manner, who are developing various types of advanced non-light-water reactors.

Griffin is a MOOSE-based reactor physics code for multiphysics applications, including steadystate and transient radiation transport, core performance, fuel depletion, etc. Developed in a consistent multiphysics environment, Griffin currently possesses the capabilities of MAMMOTH/Rattlesnake and is adopting the capabilities of PROTEUS that are needed in the code. The purpose of this document is to identify the requirements for the Griffin application and to propose a development plan for the implementation of the capabilities needed to satisfy the requirements.

Section 2 presents a code release schedule. In Section 3, we list overall and reactor-specific functional requirements for Griffin. The sections that follow, Sections 4 10, provide a more detailed description and scheduling information of the required capabilities. Finally, a condensed table format of the Griffin capabilities can be found in Appendix A. 


\section{RELEASE SCHEDULE}

The initial production version of Griffin will be released in FY 22, which will be followed by updated versions at the end of every fiscal year. The version numbers are labeled in accordance with procedure PNL-4005 [7], with a tag based on the date of release ("YYYY-MM-DD"). If a release is altered via a corrective action, the tag for the patched version shall include a patch number as "YYYY-MM-DD-pN", where " $\mathrm{N}$ " is the patch number.

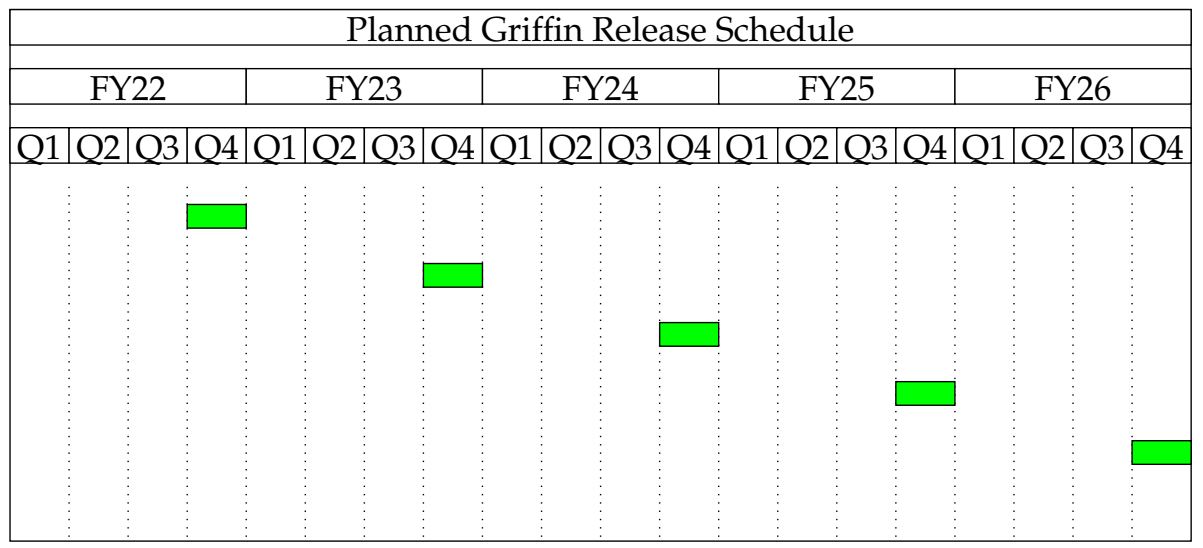

\section{FUNCTIONAL REQUIREMENTS FOR REACTOR PHYSICS}

Griffin is developed for use in the design and analysis of advanced non-light-water nuclear reactors, which represent various types of existing and future designs, including sodium-cooled fast reactor (SFR), Lead-cooled fast reactor (LFR), high-temperature reactor (HTR), pebble-bed reactor $(\mathrm{PBR})$, fluoride high-temperature reactor (FHR), molten-salt reactor (MSR) as well as microreactors. Griffin is developed on the MOOSE framework to facilitate multiphysics coupling with other physics tools for steady-state and transient conditions. The code is developed mainly with $\mathrm{C}++$, currently maintained on the INL HPC Gitlab repository, and follows processes delineated in the MOOSE development standard as well as the NQA-1 standard [8]. The key capabilities required for a reactor physics code are discussed in this section.

Reactor physics codes must meet a set of general requirements to provide the basic functionality for reactor physics applications. Section 3.1 lists the general functional requirements for Griffin with a brief description of their purpose. In addition, there are various data and calculation workflows that are specific to a reactor type. These specific workflow requirements are discussed in 
Section 3.2

\subsection{General Requirements}

The requirements listed herein are common to most reactor types and constitute the basic capability of any reactor physics code.

Neutron and photon cross-section, heating, damage, and gamma-production data - Griffin will support the preparation of cross sections using $\mathrm{MC}^{2}-3$ [1] and the cross section application programming interface (CSAPI] [9] solver interfaces. The cross-section preparation can be deployed in a traditional two-step method (lattice-core calculations) or online (i.e., executed during the core transport calculation). The cross-section data is handled by the ISOXML module in Griffin, which currently supports reading and converting Serpent2 [10], ISOTXS [11], AMPX [12], and ACE [13] formats. The nonlocal energy deposition from photons can be handled in two ways in Griffin: 1) implicitly, via the "EnergyDeposition" cross section in the neutron ISOXML library or 2) explicitly, with the "HeatingFactor" cross section in the photon ISOXML library, which is later used in a coupled power calculation. In addition, damage energy (used in the computation of displacements per atom (DPA) ) and gas production cross sections will be also available to study effects on materials.

Geometry, mesh and material assignment - The geometry definition and mesh can be generated with the MOOSE mesh generator system or with external tools, such as CUBIT [14]. The core mesh will include spatial identification variables to define unique material, equivalence, depletion, and reporting zones used in the reactor physics calculations. The addition of other spatial identification zones will also be considered, for example, in the case used to restrict variable transfers based on spatial identification zones where energy conservation is enforced with inconsistent meshes. A hierarchical system will be implemented, that allows the definition of assemblies and pins for depletion and reporting purposes. Griffin will also consider special partitioners to improve parallel scalability on unstructured grids with the current discrete ordinate $(\mathrm{SN})$ sweeper.

Input and output system - Input and output systems are essential in providing necessary input options to software and producing outputs necessary for nuclear core design and analysis. The 
Griffin input format is consistent with the MOOSE syntax. The MOOSE action system provides the means to simplify the input file structure; thus, it is widely used in Griffin. The code still retains full access to all classes for advanced users. MOOSE supports a number of output file formats in the "Outputs" block (Exodus, csv, etc.). Additional output classes are currently available in Griffin to generate engineering style maps. These will be integrated with the region identification variables to produce various levels of reporting. Significant work is needed in this area to support various reactor types and provide users with flexibility.

Eigenvalue and fixed-source calculations - All Griffin production transport solvers will support solutions to the fixed source and k-eigenvalue problems. Multiple solvers are provided for users to select the best solver for a problem in terms of performance and accuracy. Diffusion, discrete ordinate $(\mathrm{SN})$, and spherical harmonics $(\mathrm{PN})$ based on a Discontinuous Finite Element Mesh (DFEM) or Continuous Finite Element Mesh (CFEM) are currently available, and the Variational Nodal Method (VNM) [15] and the Method of Characteristics (MOC) [2] are being included.

Transient calculations - All Griffin production transport solvers will support space-time kinetics. The improved quasi-static (IQS) method [16] will be available to accelerate transient calculations. In addition, a point kinetics equation (PKE) solver is currently available. The dynamics of xenon-induced power oscillation will be handled for reactor control and stability analysis.

Depletion and fuel cycle analysis - Macroscopic and microscopic depletion options will be available. The user is able to provide custom depletion chains in the ISOXML format for microscopic depletion (decay constants, fission yields, branching ratios, Q-values, etc.). The depletion and transmutation chains as well as the fission yields generated with Griffin will be consistent with the cross sections. Nevertheless, the Griffin distribution includes a default ISOXML file with depletion chains for about 300 isotopes [17]. A reduced microscopic depletion model will be developed, including the chains of xenon, samarium, and burnable absorbers explicitly (especially for gadolinia and erbium) for an accurate estimation of number densities with depletion. These latter depletion models are reactor-design specific.

In addition to depletion, the shuffling and reloading of fuel will be added to support fuel cycle calculations and in-core fuel management, which requires the tracking of isotopic inventories of 
all fuels in successive cores. Griffin will provide the ability to track fission product poisons with the capability to

- Decay short-lived and saturate long-lived fission products poisons, which are used in fuel cycle analyses during shutdown/shuffling,

- Calculate the steady-state concentration of fission product poisons, which are used in depletion analyses.

- Perform an equilibrium cycle calculation required for fast reactors (e.g., REBUS-3 [18]) and useful for other reactor types to efficiently determine burn cycle time, control requirements, fuel enrichments, and general system performance characteristics.

Decay heat - Griffin will support three approaches for the decay heat calculation that allows for a $3 \mathrm{D}$ representation of the decay heat distribution.

- User-provided time-dependent fractional decay heat function (decay heat power divided by the steady-state power), which is used to compute the local decay heat contribution with a multiplier.

- Local decay heat power based on the ANSI/ANS-5.1-2014 standard. This approach relies on a user-provided fractional power data file for the isotopes: ${ }^{235} U,{ }^{238} U,{ }^{239} \mathrm{Pu}$, and ${ }^{241} \mathrm{Pu}$.

- Explicit calculation of the decay heat from the microscopic depletion.

Core design capabilities - Features required for use in code design will be in place.

- Management of control rods or drums. Support time-dependent unidirectional (x,y, or z) movement of materials in the computational mesh as well as independent drum rotation. High-order spatial cross-section reconstruction approach to treat the cusping effect [19].

- Automatic generation of design and safety parameters required for nuclear design, physics tests: excess reactivity, shutdown margin, reactivity coefficients, control rod worth curves (integral and differential), power offset, poison worth, radial and axial peaking factors, kinetic parameters, etc.

- Criticality search using control rods or absorber materials (e.g., soluble boron). This capability needs to be extended to support MOOSE subApps. 
- Perturbation, standard and generalized, and sensitivity calculations for safety and uncertainty analysis.

Coupling with other physics tools (under the MOOSE framework) - Multiphysics coupling is essential to the actual simulation of reactor problems and validation of Griffin, which is naturally taken care of by the MOOSE system as long as Griffin is developed under and in compliance with the MOOSE framework. Per user request, a loose or tight coupling option is available in the MOOSE system.

Verification and validation - "Verification is the assessment of the accuracy of the solution to a computational model" [20]. The Griffin development procedure integrates the verification of the various computational methods with models of varying complexity through unit testing, integration testing, system testing, and user acceptance testing. "Validation is the assessment of the accuracy of a computational simulation by comparison with experimental data" [20]. Since validation tests are dependent on a specific target system, these tests here are limited to general and common tests of capabilities and features for reactor types (SFR, MSR/FHR, HTR, PBR, and microreactors). Primarily, relevant validation benchmark problems available from the ICSBEP and IRPhEP [21] handbooks will be utilized. Additional code-to-code comparisons will be developed as needed to fill gaps, but those cannot be considered validation benchmarks.

\subsection{Reactor Type Specific Requirements}

In addition to the general requirements addressed in the previous section, further items need to be accounted for to address the needs specific to a reactor type. In this section, additional items required for SFR/LFR, MSR/HFR, HTR and microreactors are discussed.

\subsubsection{Sodium or lead-Cooled Fast Reactor}

The following characteristics should be accounted for in SFR/LFR design and analysis.

\section{Cross-section generation}

- Whole core spectrum and specially spectrum transition from fuel to reflector 
- Anisotropic scattering affecting core leakage as well as region-to-region neutron traveling

- $(n, 2 n)$ and $(n, 3 n)$ reactions are non-negligible, and inelastic scattering and unresolved resonances are important

- Scattering resonances of intermediate atomic weight nuclides (sodium, oxygen in the case of oxide fuel, and structural materials like iron, chromium, nickel, etc.) give rise to the strongly jagged structure characteristic of fast reactor spectra, resulting from the energy self-shielding phenomenon

- Doppler effect of neutron fission and absorption cross sections in the keV energy range.

\section{Thermal expansion and assembly bowing}

- Radial and axial expansion - Due to thermal expansion, irradiation-induced swelling, and irradiation-enhanced creep. Effect of thermal expansion of oxide/metal fuels in the cladding tube

- Control rod driveline expansion - Due to difference in thermal expansion of control-ride driveline and reactor vessel

- Assembly bowing - Reactivity changes due to the assembly bowing should be properly computed by deforming the mesh structure, and additionally accurate assembly duct heating is required to evaluate the bowing effect of assemblies.

\section{Coolant density and void worth}

- Effect of changes in coolant atom densities, including voiding.

\section{Additional characteristics}

- Wrapper tube

- Normal temperature range of $400-600^{\circ} \mathrm{C}$ and relatively large temperature increase of about $150^{\circ} \mathrm{C}$

- High neutron flux of about $5 \times 10^{15} \mathrm{\#} / \mathrm{cm}^{2} \cdot \mathrm{s}$

- Large neutron leakage of about $25 \%$

- Duct in fuel and control assemblies (orifice): single duct for fuel assembly and double ducts for control assembly. 


\section{Transient simulations of interest}

- unprotected loss of flow (ULOF)

- unprotected loss of heat sink (ULOHS)

- unprotected transient overpower (UTOP

- Unprotected station blackout.

\subsubsection{Molten-Salt Reactor}

The following characteristics should be accounted for in a MSR design and analysis. MSRs could be fast or thermal reactors depending on the design.

\section{Characteristics}

- Flowing fuel

- Time-dependent delayed neutron precursor advection where the neutronics and fluids are tightly coupled

- Online reprocessing and refueling

- Corrosion

- Structure/reflector feedback

- Radiation heating of reflectors and other in-vessel components

- Cross sections (e.g., chloride) for various molten salts

- Isotope species tracking (with interactions bubble, off-gas, depositions, etc.).

\section{Transient simulations of interest}

- ULOF-positive reactivity

- Unprotected single-pump failure in a multi-circuit

- Control system response time to prevent freezing in heat exchanger

- Continued operation with one or more failed pumps

- Core behavior due to inlet flow and temperature asymmetry

- Multi-circuit instabilities and assess impact on stability of core power distribution. 


\subsubsection{High-Temperature Reactors with Static Fuel}

The following characteristics are dominant for high-temperature reactors with prismatic type fuels. Mostly Tristructural Isotropic (TRISO) particulate fuels are used for an HTR because of its structurally good resistance to high temperature. A gas (helium or carbon dioxide) is used as a coolant because of high-temperature heating for high thermal (plant) efficiency.

\section{Characteristics}

- Axial streaming from gaps and large control rod hole, and large penetrations for shutdown systems - use of anisotropic diffusion

- TRISO particles in compact

- Double heterogeneity within the fuel blocks/elements

- Graphite cross sections and impurity

- Gamma heating important for reflector zones and structures

- Depletion of burnable poisons in fuel blocks and control blocks in reflectors.

\section{Transient simulation of interest}

- depressurised conduction cooldown (DCC) or depressurised loss of forced cooling (DLOFC) with/without reactor trip

- pressurised conduction cooldown (PCC) or pressurised loss of forced cooling (PLOFC) with/without reactor trip

- Load following (power maneuvering)

- Reactivity insertion from moisture (water) ingress with reactor trip

- Prompt critical reactivity insertion from water ingress with reactor trip

- Reactivity insertion by control row withdrawal

- Reactivity insertion by control row ejection

- Reactivity insertion from cold coolant injection

- Xenon stability test (axial Xe oscillations). 


\subsubsection{Pebble-Bed Reactor}

Pebble depletion is the computation of the isotopic composition of pebbles as they move through the core, are recirculated from the core outlet to the core inlet, and finally discarded. Pebble shuffling is a subtask within the pebble depletion computation. The pebble shuffling routine tracks pebbles (not isotopic content) of a certain type as they move along through the core and are mixed at the core outlet, separated into discarded and recirculated pebbles, mixed with fresh pebbles, and finally reloaded into the core. In addition to pebble shuffling, the pebble depletion requires capabilities including neutron and gamma flux solvers, "online" cross-section generation, transmutation, and a decay solver. A pebble shuffling capability is necessary for pebble depletion calculations. Pebble motion in a pebble-bed reactor can be explicitly modeled or treated as an incompressible fluid (streamline method).

Griffin will include two methods for computing flux, power, and isotopic distributions during the depletion of a pebble-bed reactor:

- A low-resolution approach that relies on the streamline method [22, 23]. This analysis capability is well suited for fuel cycle studies and trade studies

- A high-resolution, pebble-explicit approach using the pebble tracking transport (PTT) method [24], which is suited for the verification of the streamline method and the study of local phenomena (e.g., power peaking).

The two relevant operational scenarios for pebble depletion are equilibrium core and runningin calculations. The equilibrium core is the steady-state distribution of the various fields (isotopic, neutron/gamma flux, temperature, etc.), which is approached after operating the reactor for a sufficient duration under a consistent refueling policy (i.e., problem constraints). The running-in is the transient period that leads from a non-asymptotic core (usually the initial critical core) to the equilibrium core. The isotopic composition and related fields of the core during the running-in depend on time, whereas they are time-independent in the equilibrium core state.

\section{Transient simulation of interest}

- Same as static fuel HTRs

- Double heterogeneity within pebbles 
- Earthquake event [25].

\subsubsection{Microreactors}

Interest in compact power generation has been reemerging in recent years due to the growing demand for affordable and sustainable energy resources even in remote locations, military bases, etc. where the electricity supply is limited. A microreactor is a type of small nuclear reactor that is well suited for this purpose and has a rated power level typically under $20 \mathrm{MWth}$. The main factors required for a micro reactor are portability, flexibility, transportability, safety, selfregulability, and longevity. There are many types of microreactors, including SFR, MSR, HTR. PBR etc., possessing most of the characteristics of the corresponding reactor type. Their smaller size introduces a number of challenges with varying characteristics:

- Control drum - due to a small size, control drums are used instead of control rods

- Specialized shutdown rods

- Thermal expansion - some microreactors are composed of stainless-steel monoliths, in which thermal expansion is one of dominant contributors for reactivity changes

- Large leakage - due to a small core size, fast reactors would have large leakage $>25 \%$

- TRISO - typically for thermal microreactors

- Shielding - one of the important concerns for micro reactors

- Cross sections of yttrium-hydride moderator for thermal reactors.

\section{Transient simulation of interest}

- Heat pipe failure for a heat-pipe-cooled micro reactor

- Unexpected Control Drum rotation change

- Self-regulation and load-following

- Loss of cooling

\section{NUCLEAR DATA}

Neutron and photon cross-section data are one of the key elements required for a reactor physics code in terms of functionality and accuracy. Multigroup cross sections for fast reactors 
are typically generated using deterministic cross-section codes, like $\mathrm{MC}^{2}-3$, while those for thermal reactors are generated using deterministic or stochastic cross-section generation procedures in offline mode or cross-section application programming interface (API) in online mode. This section presents details of cross-section data processing for reactor types. The accuracy of the cross sections must be consistent with the accuracy of the rest of the physics that contribute to cross-section feedback.

The nuclear data included in the libraries:

- Neutron and photon cross sections

- Heating - both local and nonlocal

- Decay and transmutation

- Damage energy used in the computation of DPA

- Gas production cross sections.

\subsection{Fast-Spectrum Reactor}

The scattering resonances of intermediate atomic mass nuclides result in the strongly jagged structure of fast reactor spectrum, and the lack of $1 / \mathrm{E}$ spectrum for the calculation of heavy isotope resonance absorption requires very detailed modeling for slowing-down calculations. The hard neutron spectrum concentrated in the high energy range makes it important to model anisotropic scattering, inelastic scattering, $(n, 2 n)$ and $(n, 3 n)$ reactions, and unresolved resonance self-shielding. The long mean free path due to small absorption cross sections in the fast region implies a global coupling of the core and requires detailed whole-core depletion calculations. Therefore, cross sections for fast reactors should be treated differently than those for thermal reactors. $\mathrm{MC}^{2}-3$ has been widely used for multigroup cross sections of fast reactors. Using $\mathrm{MC}^{2}-3$, broad group (BG) cross sections are generated with two major steps.

- Step 1 - Generate ultrafine group (UFG cross sections for homogeneous or 1D calculations for assemblies, using which a core calculation is performed for a $\mathrm{R}-\mathrm{Z}$ equivalent core to generate region-wise UFG flux and moment solutions,

- Step 2 - Generate broad group (BG) cross sections for homogeneous or 1D calculations for assemblies, which is basically the same calculation but condenses UFG to BG cross sections using the region-wise UFG flux and moment solutions provided from Step 1. 
TWODANT [26] or PARTISN [27] has been used for a R-Z calculation, but due to the licensing requirement for those codes, a substitute code may be needed to make its licensing together with Griffin easier. A substitute code is required to meet the following conditions: running fast with a UFG cross section to produce flux and higher moment solutions, being capable of parallel processing, handling R-Z, and if possible, R- $\theta-Z$ geometries. Note that, in the two-step approach for cross-section generation, approximations are involved in determining 1D assemblies and an $\mathrm{R}-\mathrm{Z}$ core equivalent to actual assembly and core geometry configurations, respectively. The approximations may affect the accuracy of resulting BG cross sections. Alternatively, a Monte Carlo code has been used to generate multigroup cross sections, and while Monte Carlo-generated cross sections for thermal spectrum reactors work reasonably well, those for fast spectrum reactors have shown large errors due to an inaccurate estimation of anisotropic scattering whose effects are significant in fast spectrum reactors. In addition, the uncertainties of tallied cross sections in the very high and very low energy range of the BG energy structure are large even though their effects are insignificant.

\subsection{Thermal-Spectrum Reactor}

In thermal reactors, the neutron mean free path is typically $1-2 x$ a fuel pin diameter. Therefore, local neutron spectra are relatively independent of a core configuration. Conventionally, a twostep approach is used to generate homogenized assembly-based cross sections, in which single or color-set assemblies are used to generate fuel and control rod cross sections and a multi-assembly configuration is employed to obtain reflector cross sections. Equivalence parameters, such as assembly discontinuity factors, are generated at the same time to preserve neutron currents between assemblies with reference heterogeneous geometry conditions. The pin power distribution can be optionally reconstructed by applying pin form factors. For heterogeneous geometry problems, the cross-section application programming interface (CSAPI) [9] is used to generate multigroup cross section on the fly using the whole-core geometry and material conditions. The CSAPI is able to support the following three cross-section method options, among which the third option is being implemented.

- The subgroup method [28] - Fluxes and resonance integral are represented with subgroup 
parameters (levels and weights) in the quadrature form. The accuracy of the subgroup method relies on the accuracy of the subgroup parameters and the consistency in the enforced equivalence relation. The subgroup weights are determined using the least square approach to minimize the errors in the reconstructed effective cross sections compared to many heterogeneous pin cell reference solutions.

- The resonance integral (RI) table method - Reference multigroup cross sections are generated from a deterministic neutron slowing-down code or a Monte Carlo code based on representative pin cell calculations. Those cross sections are correlated with the corresponding RI table that is function of background cross sections derived from the deterministic code for each isotope. In the current procedure, OpenMC [29] or Serpent2/NJOY [30] is used for the reference microscopic cross section generation, and $\mathrm{MC}^{2}-3$ with the $2 \mathrm{D} \mathrm{MOC}$ capability is employed for calculating the corresponding background cross sections.

- The equivalent Dancoff-factor cell (EDC) method [31] - Region-dependent multigroup cross sections are calculated by performing a hyperfine group (HFG) slowing down calculation in a one-dimensional (1D) cell that preserves the Dancoff-factor of each pin (or each pebble). Each axial segment of every fuel pin in a lattice (or each pebble) is decoupled to 1D cylindrical (or spherical cell), which preserves the Dancoff factor at the black fuel limit. The Dancoff factor for an axial segment of a fuel pin (a pebble) is calculated using the enhanced neutron current method (ENCM) [32] that only requires a one-time fixed source calculation. Then, an equivalent 1D cell for an axial segment of a fuel pin (a pebble) is determined by the binary search, which seeks the outer radius of the cell giving the same Dancoff-factor from the 1D collision probability method (CPM). Once the outer radius of the $1 \mathrm{D}$ cell is determined, the HFG slowing down calculation is performed to obtain the HFG weighting spectrum used to obtain multigroup cross sections.

Conventional high-temperature gas-cooled reactors (HTGRs), pebble-bed reactors (PBRs), and many recent microreactor concepts use TRISO (Tristructural Isotropic) fuel grains embedded in a ceramic or metal matrix. These particulate fuels are characterized by the double heterogeneity caused by randomly dispersed fuel particles in fuel compacts or pebbles which are heterogeneously arranged in the core. To accurately generate multigroup cross sections for such cores, the double heterogeneity effect should be accounted for by proper energy and spatial self-shielding 
of resonances.

- The William's [33] and Sanchez-Pomraning [34] methods - These methods are implemented in $\mathrm{MC}^{2}$-3. The William's method in SCALE employs a two-step procedure in which compactaveraged cross sections are computed using the flux-volume weighting at the first step and a lattice self-shielding calculation (hyperfine group slowing down calculation) is performed using compact-averaged cross sections at the second step. The Sanchez-Pomraning method in APOLLO-II directly performs the fuel compact lattice calculation with the cross sections of each layer of fuel particle and those of matrix in a fuel compact using the method of characteristics (MOC) modified.

- The iterative local spatial self-shielding (ILSS) approach [35] - This method, implemented in $\mathrm{MC}^{2}-3$ as well, provides more accurate solutions than the two existing methods above. The overall procedure is the same as that of the Williams method, but the way to calculate the disadvantage factor is different. The ILSS method accounts for the effect of randomness of particles on spatial self-shielding inside particles. The composition of the outside of the particle is a homogenized compact (particles + matrix), of which cross sections are spatially self-shielded. Since the spatial self-shielding condition is determined after solving the particle cell problem, disadvantage factors are determined iteratively until the solution converges.

The ILSS method is available in $\mathrm{MC}^{2}-3$ to generate cross sections for the TRISO fuel in the conventional way (two steps). The method is being implemented in CSAPI so that the TRISO fuel can be handled in the cross section generation online. In the implementation to CSAPI,

- The first level of heterogeneity (the TRISO level) is treated by the ILSS method

- The second level of heterogeneity (the lattice level) is treated by the equivalent Dancoff factor cell (EDC) method.

\subsection{Equivalence Parameters}

Diffusion is used as the main solver for quick core calculations; however, improved accuracy with diffusion relies on the use of homogenization equivalence to preserve the reaction rates in the core. The calculation of discontinuity factors (DFs) [32] is available with the discontinuous 
transport solvers given surface fluxes/currents, whereas the calculation of superhomogenization (SPH) [36] factors is available for CFEM solvers given volume-integrated fluxes. Furthermore, Griffin includes a hybrid DF-SPH method for DFEM solvers. The INL ISOXML standards currently support the addition of an equivalence data library for the various equivalence techniques. SPH factors are generated for applications to a core problem with homogenized fuel pins or assemblies. Assembly discontinuity factor (ADF) or directional diffusion coefficients may be used together with SPH for fuel assemblies (FAs) and reactor core boundary regions to preserve FAwise reaction rates and/or neutron leakage of the core. Since cross-section generation and core calculation are performed based on homogenized fuel assemblies, pin information needs to be reconstructed by superimposing the calculated homogeneous solutions with the precalculated pin form function of the fuel assembly.

\subsection{Representation of Cross Sections and Equivalence Data}

Griffin currently uses a traditional tabulation approach in which the cross section or equivalence table is interpolated based on the local values of the tabulated variables. The interpolation resolution should be adaptable and depend on an integer variable defined in the geometry definition. This will allow users to interpolate cross sections at element, pin, and assembly level, depending on the level of multiphysics fidelity that is required for the calculation. Research is needed in the area of multivariate data representation to speed-up the cross-section interpolation, regression, and sparse-grid approach with decomposition. This is specially necessary in the context of microscopic depletion at a finer scale (pin-by-pin).

\subsection{Cross-Section Library Generation}

For the $\mathrm{MC}^{2}-3$ approach, neutron and gamma cross-section libraries should be provided, which include isotopic data for UFG principal cross sections, resonance data, prompt and delayed gamma data, heating data, etc. For the CSAPI approach, cross-section libraries need to be generated using OpenMC (or Serpent2/NJOY) and $\mathrm{MC}^{2}-3$ calculations, which include isotopic data for UFG principal cross section, resonance parameters as a function of temperature and background cross section, heating data, etc. 


\subsection{Tasks and Schedule}

Preliminary cross-section capabilities are currently available or will be ready in FY-21. The items and timeline for advanced cross-section capabilities are as follows:

1. Set up the two-step and online cross-section generation workflows for fast and thermal spectrum systems.

2. Generate neutron and gamma data libraries for specific reactors or reactor systems and develop a procedure for the library generation.

3. Optimize the broad group cross-section structure and develop a procedure for the optimization.

4. Improve the cross-section data parameterization algorithm in terms of performance and data storage.

5. Improve the performance of a whole core calculation using coarser group cross sections while an online cross-section generation is performed with finer group cross-section structures.

6. Improve the accuracy of anisotropic multigroup cross sections accounting for directional changes of the anisotropic effect.

7. Include the double heterogeneity effect in the cross-section generation to deal with particulate fuels in a core.

8. Allow simplified or detailed nuclide decay chains depending upon users' needs in terms of performance vs. accuracy.

9. Update cross-section libraries with ENDF/B-VII and VIII data.

10. Support data for transient calculations as well as large calculations. 


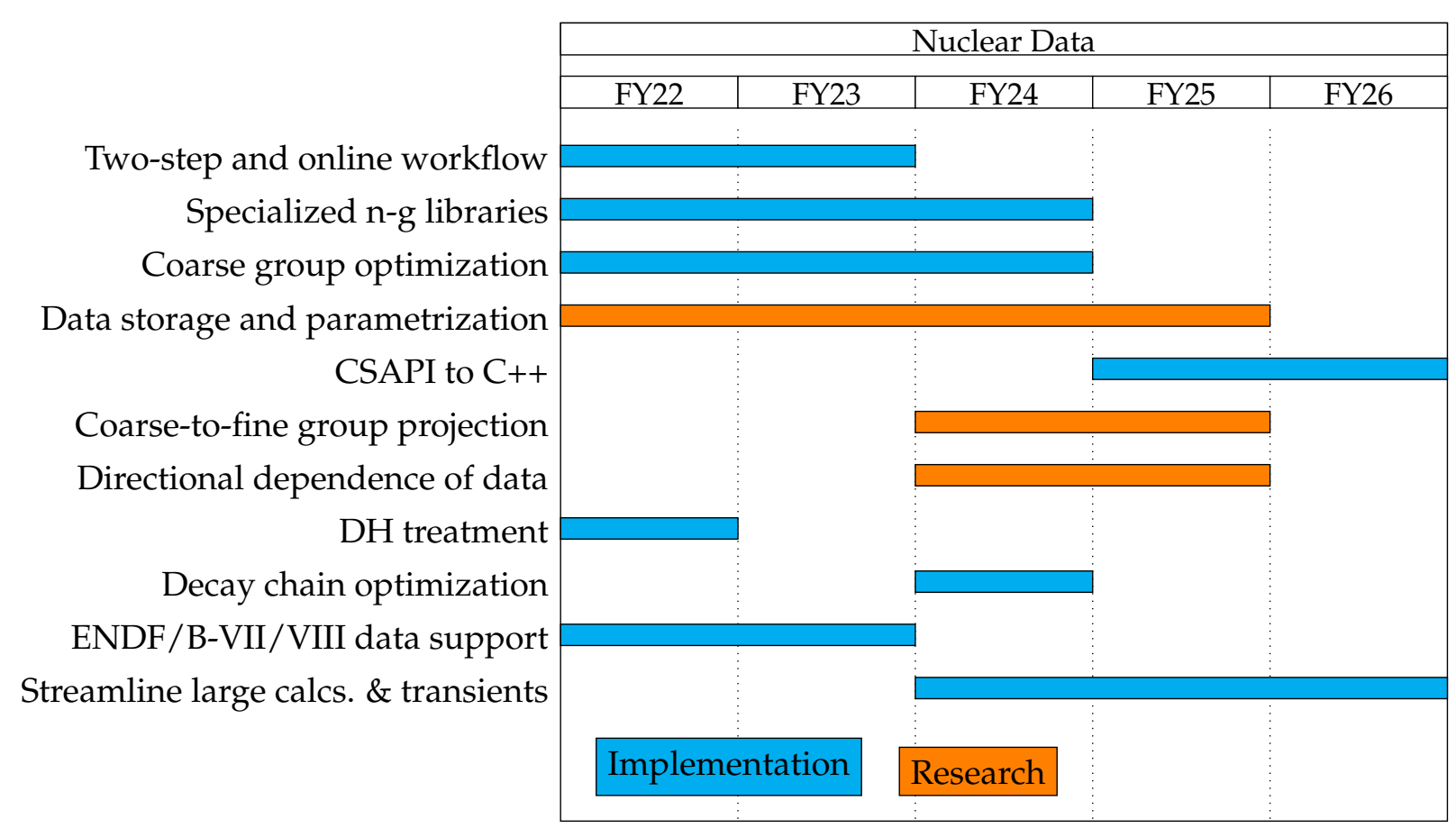

\section{GEOMETRY}

Griffin is based on the libMesh [37] library, which provides a framework for the numerical simulation of partial differential equations using an arbitrary unstructured discretization on serial and parallel platforms, supporting 1D, 2D, and 3D steady-state and transient simulations on a variety of popular geometric and finite element types. This section presents details of regular and unstructured mesh that will be supported by Griffin. A meshed geometry can be constructed by external mesh generation tools, such as CUBIT, and the mesh generation capabilities for Cartesian, hexagonal, and regular triangular structured geometry should be available in the MOOSE system to make it easy to construct mesh files with reporting IDs based on the hierarchical structure (e.g., pin, assembly, plane, and core), which facilitate identifying where each mesh belongs to. Once a meshed geometry is built, materials and cross sections need to be assigned to regions that are determined during the meshing process. In the code, IDs listed below are set based on the inputs from a mesh file and a user input file.

- Element ID - every element 
- Reporting ID - every element that includes hierarchical structure information, such as pin ID, and assembly ID

- Subdomain ID - a requirement of the Exodus II format [38], also called block ID, a group of elements neighboring each other with the same material

- Material ID - every material region

- Cross section ID - every region that have unique cross section data sets. The same material in a different region is defined as a different cross section ID

- Depletion ID - every cross section ID with different number density history

- Equivalence ID - every SPH or GET region that includes a single material.

The relationships between IDs are as follows: the same material can be categorized in different cross-section groups especially when the regions are separate from each other, and the same cross section groups can be divided into different depletion regions when depletion and resulting isotopic number densities are noticeably different with time, affecting the cross-section self-shielding. The reporting ID allows us to edit outputs on the pin and assembly basis. A mechanism will be developed to rename ID regions in a given Exodus output file so that the user can postprocess results. This situation can arise if the user wants to change the reporting resolution but keep the same solution in the Exodus file.

Regular geometry - Cartesian and hexagonal type of meshes in a homogeneous or heterogeneous configuration can be generated using the MOOSE mesh generators. Meshes in the Exodus format can be provided from external meshing tools, such as the Argonne Mesh Tool [39] and CUBIT [14]. A fine (regular) mesh for a higher-order calculation (SN, PN, and MOC) and a coarse mesh for the solution convergence acceleration with nonlinear diffusion acceleration (NDA) or diffusion synthetic acceleration(DSA) should be consistent with each other.

Unstructured geometry - a mesh is normally generated by external meshing tool such as CUBIT. In this case, it may be difficult to generate a coarse mesh equivalent to a fine (regular) mesh 
for the acceleration with NDA or DSA. We may need to introduce the coarse-mesh finite difference CMFD approach in which a polygonal-type coarse mesh can be determined automatically based on the fine mesh. However, since MOOSE does not currently allow polygonal meshes, the approach will be considered when MOOSE is updated in future.

Pebble Tracking Transport (PTT) [24] Geometry - Each individual pebble is tracked and modeled on an unstructured mesh whose nodes correspond to the pebble centroids. The neutron transport equation is solved on this pebble tracking mesh with pre-assembled elemental matrices. These elemental matrices are independent of the cross sections and the streaming directions and they can be assembled before the calculation without introducing an excessive memory overhead, especially when combined with spatial domain decomposition.

Coarse mesh (CM) generation for $\mathrm{CM}$ acceleration - A CM will be generated to be consistent with the fine mesh in order to support the coarse mesh acceleration with the NDA, DSA, or CMFD methods.

Schedule - Assuming that the main meshing capability is provided from the MOOSE framework, the following tasks will be focused in Griffin.

1. Use the hierarchical structure of IDs available for efficient cross-section manipulation and input/output edits.

2. Support the advanced MOOSE mesh generators for reactor geometries and acceleration methods.

3. Support the mesh displacement.

4. Support the PTT calculation for PBR design. 


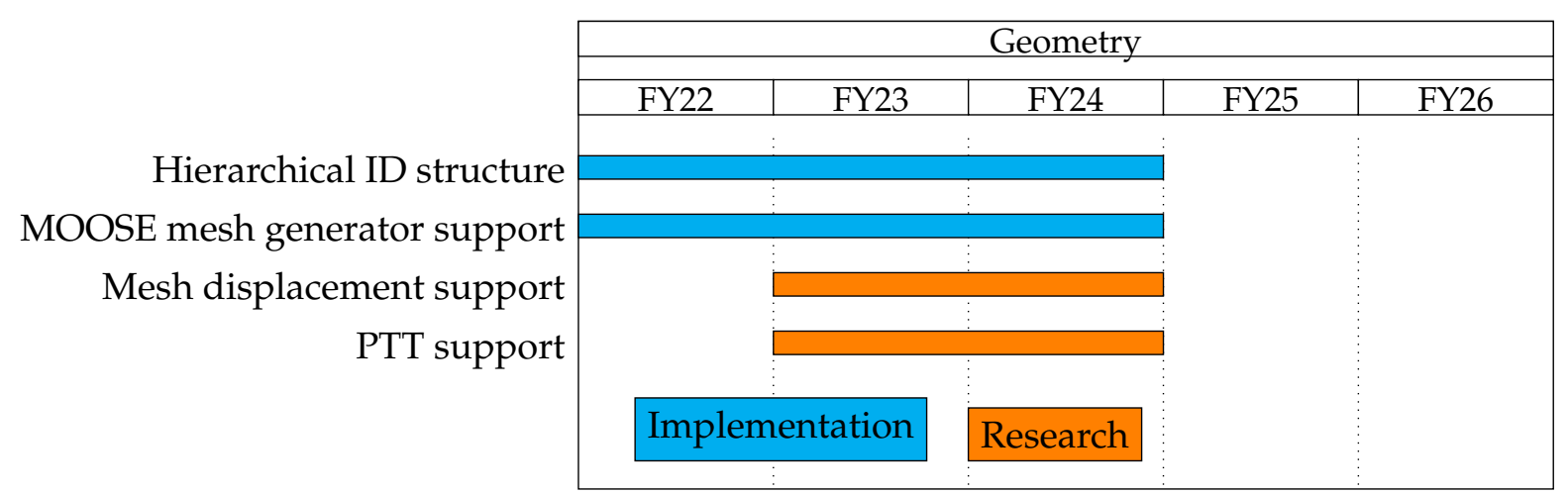

\section{INPUT AND OUTPUT FILE STRUCTURE}

This section describes the ideal input structure for Griffin and the recommended output for specific calculation.

Input file structure - The Griffin input format is consistent with the MOOSE syntax. Griffin relies on the MOOSE action system to simplify the input file structure but retains access to all classes for more advanced users. The Griffin code development standards do and will continue to emphasize the use of the easy, consistent, logical, and flexible structure of the input blocks and associated parameters for steady-state, transient, depletion, fuel cycle calculations as well as output editing for coupled calculations and downstream codes. Griffin uses ISOXML-formatted data for the multigroup nuclear and the decay and transmutation data. The ISOXML module is included in Griffin and allows users to convert specific formats, like ISOTXS and AMPX, to the ISOXML format. Significant work is needed in the ISOXML module to improve the documentation and support for users. Given the flexibility in MOOSE, it is possible to automate standard reactor physics calculations.

Output file structure - MOOSE supports a number of output file formats in the "Outputs" block (Exodus, csv, etc.). The Exodus format output can be visualized in VisIt [40] and Paraview [41]. Additional output classes are currently available in Griffin to generate engineering edits. These engineering edits, in text format, will be added for standard regular geometries (Cartesian, cylindrical, and hexagonal). In addition, special edits will be integrated with the region identification variables included in the mesh to produce various levels of reporting (pin ID and assembly ID). 
Typical outputs include scaled fluxes (corrected for SPH factors), power distributions, and other reaction rate distributions. In addition, feedback quantities can also be reported in these edits (temperatures, density changes, displacements, etc.) Outputs are required for storing depletion information for a physical entity, potentially at the pin level, since in some reactors, pins can be moved into other assemblies. The output files must be compressed and should include the pin history (flux, fluence, energy, damage, isotopic composition, etc.). Significant work is needed in this area to support various reactor types and provide users needed flexibility. HDF5 [42] is a good candidate since this data format that is commonly used in production codes.

User interface with Workbench - PyGriffin [43] in Workbench [44] will support users to process inputs and outputs as well as run jobs. The tool will help visualize problem geometry, composition assignment, mesh, cross sections, etc.

Data post-processing - Griffin will support various post-processing tools that can streamline generation of essential reactor physics outputs, such as pin power, core spectrum, peaking factors, etc. An appropriate compressed data format HDF5 [42] will be needed.

Tasks and Schedule - The preliminary input and output systems are currently available or will be ready in FY-21. The input and output systems will continue to be improved to support users' needs.

1. Improve the documentation of the ISOXML format by updating the multigroup, decay, and transmutation data structure. Improve the ISOXML module documentation of the various methods and examples for use

2. Add automated calculations for data auditing capability, run equivalence state points, control rod worth calculations, reactivity coefficients, etc.

3. Prepare a convergence table that can help pinpoint specific problems in the convergence behavior

4. Add pin edit capabilities for heterogeneous or pincell homogenized geometries

5. Add pin reconstruction for assembly homogenized geometries

6. Edit in-core and ex-core detector responses 
7. Add engineering edits for core distributions (flux, power, reaction rates, etc.) in regular geometries

8. Support Workbench/PyGriffin

9. Support Visualization

10. Automate error checks.

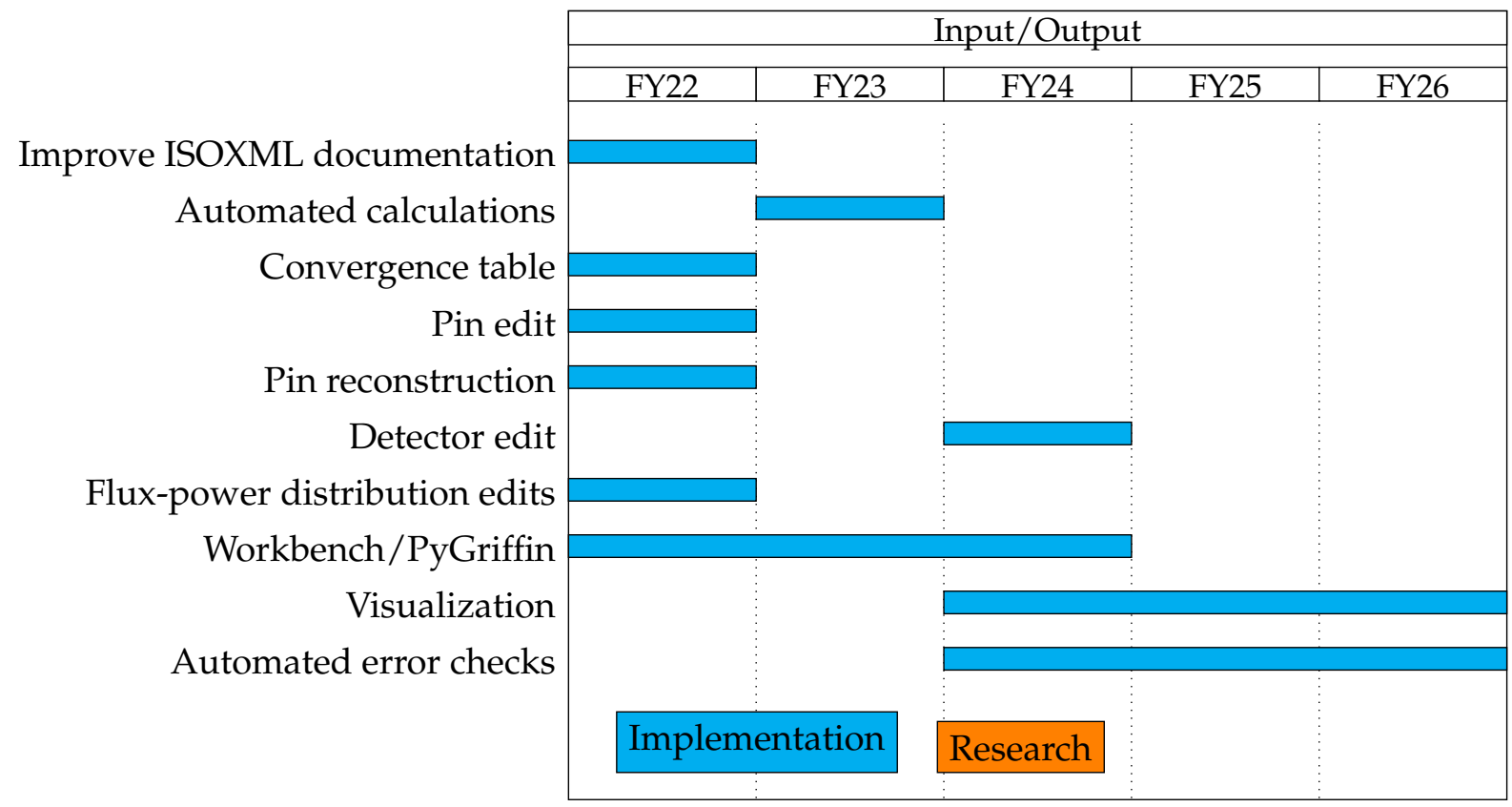

\section{CALCULATION CAPABILITIES}

This section specifies the calculation capabilities of the Griffin application. Section 7.1 details the transport solvers and methodologies that interact with the Griffin transport system. A discussion on the fuel cycle needs follows in Section 7.2. Requirements on perturbation/sensitivity and uncertainty quantification capabilities are proposed in Section 7.3. Finally, Section 7.4, discusses future integration efforts with the Shift code.

\subsection{Transport Solvers}

Griffin incorporates a number of Boltzmann transport equation solvers for a variety of particles including neutrons, thermal radiation (i.e., photons of $0.1-1,000 \mu \mathrm{m}$ wavelength), gamma radiation (high energy photons), phonon, and charged particles (e.g., electrons, protons, alpha 
particles). The type of equation to solve can either be the primal transport equation with the particle flux as the solution variable or the adjoint equation with the adjoint or importance flux as the solution variable. All equations are developed in a multigroup formalism, requiring specification of multigroup cross sections as input. Three different coordinate systems available are: 3D Cartesian (X-Y-Z), 2D cylindrical (axial-symmetric or R-Z), and 1D spherical (R-spherical). Not all solution schemes will be consistent with all three coordinate systems. Limitations in application of different schemes for different coordinate systems will be identified in the Griffin User's Manual [45]. According to previous research outcomes and experiences, $\mathrm{SN}$ is more efficient for applications of homogeneous or mildly-heterogeneous geometry problems, while MOC works better for applications of heterogeneous geometry thermal spectrum problems. For simulating thermal expansion, SN performs better than MOC since SN runs with a fully unstructured 3D mesh while MOC works with the axially extruded mesh from an unstructured 2D mesh.

Problem Type - Five problem types will be supported, with the selection of the problem type depending on the intended application of the code:

- K-eigenvalue solves for the multiplication factor and the (self-sustained) fundamental mode in a case where no external sources of particles are present. The k-eigenvalue problem is only valid for neutron calculations. In a k-eigenvalue problem, the neutron fission sources are balanced to the losses by the inverse of the multiplication factor $1 / \mathrm{k}$. No time derivative term is present in the eigenvalue calculations. The criticality search capability will be embedded to the k-eigenvalue mode.

- Adjoint solution, where an adjoint k-eigenvalue problem is solved. Solutions to the adjoint form transport equation are useful for calculating the change in the response of a system to a small perturbation and for calculating the source that will give a desired response. The adjoint transport equation can be obtained by transposing the scattering and fission matrices.

- Steady-state fixed source solver solves a problem where the flux is driven by a distributed source and/or inflow of particle over the boundary. No time derivative term is present in steady-state calculations. The source would be input or provided by the results of a previous calculation. 
- Transient solver can comprise of distributed sources or inflow boundary conditions. Typically, those boundary conditions are provided by a previous calculation. The time-dependent precursor distribution is explicitly incorporated with the transient solver. Typically, the initial condition is provided by either a k-eigenvalue or fixed source steady-state calculation.

- IQS [16] is a time-dependent transient approximation based on the Improved Quasi-Static (IQS) approach, where temporal and spatial solutions are separated but coupled by changes in physics parameters. This problem type will also comprise of distributed sources or inflow boundary conditions, with boundary conditions provided by the previous calculation.

Solution Scheme - A given scheme will consist of the particle type, equation type, and problem type. A scheme will usually be comprised of a combination of angular and spatial discretization methods applied to a particular form of the particle transport equation. Different forms of the transport solution will include discrete ordinates (SN), spherical harmonics (PN), and diffusion approximation, implemented in a continuous or discontinuous form of FEM (i.e., CFEM or DFEM) and as a first- or second-order FEM formulation. The solution schemes available in Griffin are:

- DFEM-SN - The first-order SN formulation with DFEM. This scheme is suitable for heterogeneous problems where high angular resolution is typically required. This scheme works with fully unstructured geometries with 3D mesh displacements due to thermal-mechanical expansion. The scheme can be used for PTT calculations in PBR simulations. It can employ a matrix-free sweeping technique along with diffusion acceleration to render efficient calculations in term of of memory usage and CPU time.

- MOC (Method of Characteristics): The 2D/3D MOC based on the 2D MOC combined with the discontinuous Galerkin finite element method in the axial direction. The axial variation of angular flux and neutron source in individual computing meshes is represented with the linear (or quadratic) basis functions. This method resolves the accuracy and instability issues of the 2D/1D MOC, which relies on the homogeneous solutions to the axial direction, maintaining almost the accuracy of the full 3D MOC. Note that it is based on the axially extruded 3D mesh of the 2D mesh.

- VNM - The variational nodal method is based on a PN discretization. This scheme is efficient for homogeneous geometry-based problems with typical triangular, Cartesian, and hexag- 
onal geometry. The red-black scheme is used to improve the solution convergence. This scheme allows p-refinement; thus, coarse meshes can be used. PN angular discretization makes this method suitable for problems with pronounced streaming for fast or gas-cooled systems. This scheme supports homogenization equivalence with both DF and SPH factors.

- CFEM-Diffusion: Diffusion calculation with CFEM. This scheme works seamlessly with various PETSc preconditioned Jacobian Free Newton Krylov (PJFNK) solving options and with fine unstructured meshes. The SPH equivalence technique is available with this method.

- Additionally, CFEM-SN, -PN, and DFEM-diffusion are available.

Multi-scheme methods - The multi-scheme concept provides a capability to deploy different discretization and/or solution schemes with varying resolutions on different subdomains of the same problem without fixed-point iterations on subdomain interface variables. Regions of different levels of homogenization can be treated most efficiently with the multi-scheme approach. It can be used to avoid inaccuracies that emerge from the homogenization some heterogeneous regions (e.g., control rods). Two distinct methods are available for imposing an interface condition within the domain decomposition framework:

- The Lagrange multiplier method enforces the continuity of angular flux moments across the interface by introducing the Lagrange multipliers defined over the interface. This approach creates a saddle point problem and is only considered for CFEM schemes, such as self-adjoint angular flux (SAAF).

- The classic up-winding method has been applied at the element level in the discontinuous FEM for radiation transport and for hybrid transport algorithms. The up-winding method solves the equations for each subdomain imposing surface source boundary conditions provided from their neighbors.

Boundary conditions - The following boundary conditions are supported:

- Specular reflection

- Isotropic reflection (white)

- Periodic

- Vacuum - non-reentrant current 
- Albedo - traditional albedo matrix approach

- Time-dependent source / transport solution.

Equivalence techniques - Griffin provides a number of methods to reduce the error from energy condensation, spatial homogenization, and low spatial convergence when applying homogenization to regions of the model. The two equivalence techniques that are most commonly applied for accurate cross-section homogenization are the Generalized Equivalence Theory (GET) [46] and the superhomogenization (SPH) [36]. GET results in the generation of discontinuity factors (DFs) for each homogenized node surface. SPH methods are used to generate SPH factors which are determined for each equivalence region. Griffin will provide both options for treatment of homogenized cross sections.

Void treatment - The original SAAF formulation involves an inverse total cross-section $\left(1 / \Sigma_{t}\right)$ term, which is problematic for voids or near voids where $\Sigma_{t}$ is zero or close to zero, respectively. The SAAF-CFEM-SN scheme implemented in Griffin has a void treatment to overcome this issue.

Angular quadratures - An angular quadrature set is designed to numerically integrate a function over an angular domain and is used in discrete ordinate calculations. For each quadrature set, discrete angles and weights are derived under a different set of requirements and constraints. Those requirements typically determine the nature of the equations for which the numerical quadrature is exact. For general integration, quadrature sets have some associated error that depends on the shape of the function being integrated. Each quadrature type contains a set of angle/weight pairs for a varying number of angles; in general, the higher the order (the number of discrete angle/weight pairs), the more accurate the integration. Higher order solutions also come at a computational cost in terms of both solution speed and memory requirements. Griffin provides three quadrature sets as options for discrete ordinates calculation:

- Level symmetric - the quadrature results from the use of base points (corresponding to specific angles that are selected on the unit sphere in such a way as to preserve the symmetry of the eight octants with respect to $\pi / 2$ rotations. It may be used in $2 \mathrm{D}$ and $3 \mathrm{D}$ problems. The level symmetric quadrature set provides up to 30th order quadrature. 
- Gauss-Chebyshev or Legendre-Chebyshev - the product quadrature that uses base points derived from the roots of Chebyshev polynomials. It may be used in 1D, 2D, and 3D integration.

- Bickley-3 quadrature sets - the product quadrature derived by integration of Bickley functions.

Restart and recovery - Restart can be considered as a way of setting initial conditions with the data from a previous simulation. Griffin will support several modes of restarting:

- Restarting a stand-alone eigenvalue or transient calculation.

- Restarting an eigenvalue calculation that is used to establish the initial steady state of a transient calculation.

- Loading the transport system's variables from an Exodus file.

Griffin will support calculation recovery. Recovery is distinct from restart. Recovery is by definition resuming an existing simulation either due to a fault or other premature termination.

Acceleration methods - The following acceleration techniques are available in Griffin:

- nonlinear diffusion acceleration (NDA) - a non-linear acceleration method that is inherently effective for solving typical neutron transport problems but may only be implemented if SN is used for the angular discretization.

- diffusion synthetic acceleration (DSA - a linear acceleration method that can be used as a preconditioner for direct transport solves.

- coarse-mesh finite difference (CMFD - a non-linear acceleration method based on an unstructured coarse mesh. The base coarse mesh is currently restricted for triangular and quadrilateral shape elements, which will be extended to a general polygonal shape to deal with arbitrary geometries.

- S2 transport acceleration S2TA - an acceleration method that can be more robust for treating problems with void or near-void regions.

- Coarse Group acceleration - coarser groups are used to accelerate the convergence of the fine-group solution. 
Gamma calculation - Gamma calculation is performed to calculate gamma-induced powers.

- Read gamma yield, gamma interaction cross sections, and gamma heating data.

- Calculate gamma source distribution based on neutron flux distribution

- Perform gamma transport calculation to determine the gamma distribution that leads to the determination of the gamma-induced power distribution

Point kinetics - A point kinetics model is useful to understand the transient behavior of a nuclear reactor via changes in reactivity. Griffin is able to generate point kinetics parameters (effective generation time and effective delayed neutron fraction) as core calculation outputs, even though those parameters can be given from inputs.

Performance requirements - Deterministic calculations should run faster than equivalent Monte Carlo calculations with tallying local data required for reactor design and analysis. With the conventional fidelity calculation options, such as a diffusion calculation based on homogenized fuel assemblies, it is obvious that Griffin calculations are orders of magnitude faster than Monte Carlo calculations. For the high-fidelity calculation options, such as a transport calculation based on a heterogeneous geometry configuration, high-fidelity Griffin calculations should be done with a reasonable number of processors $(<1,000$ processors) and within 1-2 hours for a single steadystate condition. Eventually, the performance of high-fidelity calculations will be equivalent to or better than that of PROTEUS in a multiphysics environment. Note that a conventional fidelity calculation normally takes $<30$ seconds, using $<10$ processors, to solve a 3D whole-core problem with a single steady-state condition.

System and memory requirements - Assuming that 512 GB per node (80 cores per node) would be a standard configuration of a Linux machine easily available to users, about 5 GB per core would be the maximum memory limit for any size of problems. An assessment of memory usage for large depletion cases is needed. User's guidance on limits will be provided.

Tasks and Schedule - The production transport solvers and relevant systems will be ready in FY-21. Further improvements will be made for those solvers that will be available in FY-22, and additional solvers that are required for specific problems will continue to be developed. 
1. Optimize the performance of the DFEM-SN solver with coarse mesh acceleration. Improve the performance of the diffusion solver, especially in multiphysics simulations by storing the cross section data and reducing the number of interpolation calls from the materials.

2. Improve the performance of the variational nodal method with coarse-mesh acceleration.

3. Provide the R-Z or R- $\theta-Z$ fine-group solver to support the multigroup cross-section generation for fast reactor systems.

4. Complete MOC 2D/3D and allow tracking in typical reactor geometries (hexagonal and cylindrical boundaries).

5. Improve neutron- and gamma-coupled calculations for transient simulations. Allow restart and develop tutorials for coupled transient calculations.

6. Investigate the potential use of p-refinement in model convergence for various reactor types with the VNM transport solver.

7. Add linear source capability to the MOC solver,which further improves the acceleration and load balancing.

8. Enhance the DFEM-SN capability for PTT multi-zones with coarse-mesh acceleration.

9. Add hexagonal and cylindrical geometry support to libmesh to further improve the VNM solver by reducing the number of elements in the mesh and using a high order representation of the particle flux and currents.

\begin{tabular}{|c|c|c|c|c|}
\hline & & ansport Sol & & \\
\hline & FY-22 & FY-24 & FY-25 & FY-26 \\
\hline Diffusion and DFEM-SN opt. & $\square$ & & & \\
\hline VNM - acceleration & & & & \\
\hline $\mathrm{R}-\mathrm{Z}$ fine group solver & & & & \\
\hline MOC geometry tracking, 2D/3D & & & & \\
\hline Improve $n$-g coupling transient & & & & \\
\hline VNM p-refinement & & & & \\
\hline MOC lin. source, accel., load bal. & & & & \\
\hline DFEM-SN PTT multizone, accel. & & & & \\
\hline VNM - libmesh support & & & & \\
\hline S2TA & & & & \\
\hline & Implementation & Research & & \\
\hline
\end{tabular}




\subsection{Fuel Cycle Calculation}

Griffin includes microscopic depletion. The capability of reloading, shuffling and reprocessing of fuel will be added via the identification of physical zones, which correspond to assemblies, blocks, or pebbles in a core and where materials can be shuffled. A specialized data structure will be developed to store the physical zone information. The depletion materials assigned to these zones will store their historical depletion information. Additional quantities of interest, such as flux, power, local temperature and isotope inventory, will be added to the data structure.

Depletion - The depletion ID variable is used to define depletion zones. Chebyshev rational approximation method (CRAM) [47--50] and minimax polynomial approximation (MMPA) [51] are available to perform depletion based on the Bateman equation. The data requirements are introduced in Section 4 . The current predictor-corrector methodology in Griffin needs improvement and additional testing. Regular ( 300 isotopes) and reduced depletion chains will be updated or defined with lumped fission products.

Reload and shuffling - Fuel batch loading and shuffling will be based on core maps with the physical zone identification numbers. Radioactive isotopes decays during reloading intervals.

Equilibrium cycle calculation - This capability is necessary for core design and fuel management studies in various reactor types. In the equilibrium cycle calculation, the user specifies inputs, including external fuel supplies and reprocessing conditions, and the code determines the reactor burn cycle time to achieve a specified discharge burnup; the fresh fuel enrichment to achieve a specified multiplication factor at a specified point during the burn cycle; the control poison density to maintain a specified value of the multiplication constant throughout the reactor burn cycle; the reactor burn cycle time to achieve a specified value of the multiplication constant at the end of the burn step; etc. The run-in and equilibrium cycle calculations for PBRs were added to Griffin in FY-21. Additional testing and optimization is necessary to improve performance.

Tasks and Schedule - The depletion capability will be ready in FY-21. Additional capabilities and updates will be implemented as follows: 
1. Improve the depletion performance with the predictor-corrector method.

2. Improve the PBR streamline capability.

3. Provide the PTT depletion with pebble circulation for PBR.

4. Provide the capability of shuffling and refueling process.

5. Allow the equilibrium cycle calculation capability that searches for enrichment, burnup cycle length, control poison density, etc.

6. Provide additional capabilities of inventory reporting, reprocessing, and online fuel feed and removal mechanism.

7. Update the depletion capability to support the design of MSR.

\begin{tabular}{|c|c|c|c|c|}
\hline & & Fuel cyc & & \\
\hline & FY-22 & FY-24 & FY-25 & FY-26 \\
\hline Depletion P-C & & & & \\
\hline PBR streamline improvements & & & & \\
\hline PTT depletion with pebble recirculation & & & & \\
\hline Shuffling \& batch system & & & & \\
\hline Equilibrium cycle calculation & & & & \\
\hline Inventory reporting & & & & \\
\hline Reprocessing & & & & \\
\hline Depletion for MSRs & & & & \\
\hline Online fuel feed/removal & & & & \\
\hline & Implementation & Research & & \\
\hline
\end{tabular}

\subsection{Perturbation, Sensitivity and Uncertainty Quantification}

The generalized perturbation theory (GPT) method is used to calculate reactivity and sensitivity coefficients of a response parameter with respect to input parameters, such as cross sections. The reactivity coefficients are primarily used to calculate kinetics parameters for use in a safety analysis of a nuclear reactor, and the sensitivity coefficients are used to estimate the uncertainty in a given response parameter due to uncertain cross-section data. The code is able to compute reactivity coefficients and sensitivities to criticality, irradiated fuel isotopic composition, power peak, reaction rate, reaction rate ratio, and reactivity worth based upon changes in microscopic crosssection data and material density changes. The isotopic covariance matrix data associated with 
nuclear data in use are needed to predict the uncertainties of cross sections. In addition, Griffin will support forward (statistical) perturbation methods via the MOOSE stochastic tools module.

Tasks and Schedule - This capability will be implemented starting from FY-22 as follows:

1. Implement the capability of the first-order GPT.

2. Implement the capability of calculating sensitivity coefficients for cross sections and integral parameters.

3. Implement the capability of dynamic reactivity calculation.

4. Implement the capability of uncertainty calculation using sensitivity coefficients and isotopic covariance data.

5. Provide an automated process for uncertainty calculation.

6. Support for MOOSE stochastic tools module.

\begin{tabular}{|c|c|c|c|c|}
\hline & Pertı & ation and & ivity & \\
\hline & FY-22 & FY-24 & FY-25 & FY-26 \\
\hline Sensitivity coefficient & & & & \\
\hline First-order GPT & & & & \\
\hline Dynamic reactivity calculation & & & & \\
\hline Uncertainty calculation & & & & \\
\hline Automated uncertainty calculation & & & & \\
\hline MOOSE stochastic tools & & & & \\
\hline & Implementation & Research & & \\
\hline
\end{tabular}

\subsection{Integration with Shift}

The Shift [52] Monte Carlo code is currently being developed under NEAMS and will be used to generate nuclear data set for Griffin to perform multiphysics calculations.

Tasks and Schedule - This capability will be implemented starting from FY-22.

1. Griffin will write an overlay file for Shift and the necessary tallies to ensure consistency between the Griffin mesh and the Shift geometry.

2. Provide neutron source to Shift for out-core fluence and shielding calculations. 
3. Use Griffin deterministic methods to accelerate the source convergence in Shift Monte Carlo solutions for coupled multiphysics calculations.

\begin{tabular}{|r|r|c|c|c|c|}
\cline { 2 - 5 } & \multicolumn{5}{|c|}{ Shift Integration } \\
\cline { 2 - 6 } Tally consistency & FY-22 & FY-23 & FY-24 & FY-25 & FY-26 \\
\cline { 2 - 6 } & & & & & \\
Neutron source for shielding & & & & & \\
MC acceleration and coupling & & & & & \\
\hline
\end{tabular}

\section{MULTIPHYSICS COUPLING}

Griffin can be readily coupled with other MOOSE-based physics codes such as Pronghorn [53], BISON [54], Sockeye [55], SAM [56], etc. to perform multiphysics simulations. MOOSE allows strongly coupled simulations in which all physics are solved with the same mesh and system. Additionally, MOOSE also enables tightly coupled (operator splitting via Picard iteration) simulations via the MultiApp and the data transfer systems. Multiphysics support is a foundational concept for Griffin that entails the coupling of phenomena that either affect the particle transport physics or are relevant to reactor physics. In addition, this support must extend to all reactor designs. The following list includes phenomena from other physics solutions that will affect the reactor physics:

- Temperatures change - This is currently treated with the use of temperature variables (e.g., fuel, moderator).

- Geometry change - In heterogeneous geometries, this can be readily treated with the use of mesh displacement variables. For homogenized geometries, there might be additional corrections to cross sections to ensure that the underlying physics characteristics are preserved (e.g., dimensional changes of cladding geometry that might affect neutron streaming).

- Composition change - For MSR and PBR, compositions in a core can change during the operation (e.g., liquid-fueled reactor design).

Tasks and Schedule - The following items will be considered to ensure seamless multiphysics simulations with other physics tools. 
1. Support simulation of SFR cores for core expansion, sodium void and control rod worth, wrapped tube, and single or double ducted assemblies.

2. Transient support for both PBRs and HTRs with online cross sections.

3. Support transient simulations for other non-light-water reactor cores, including microreactors.

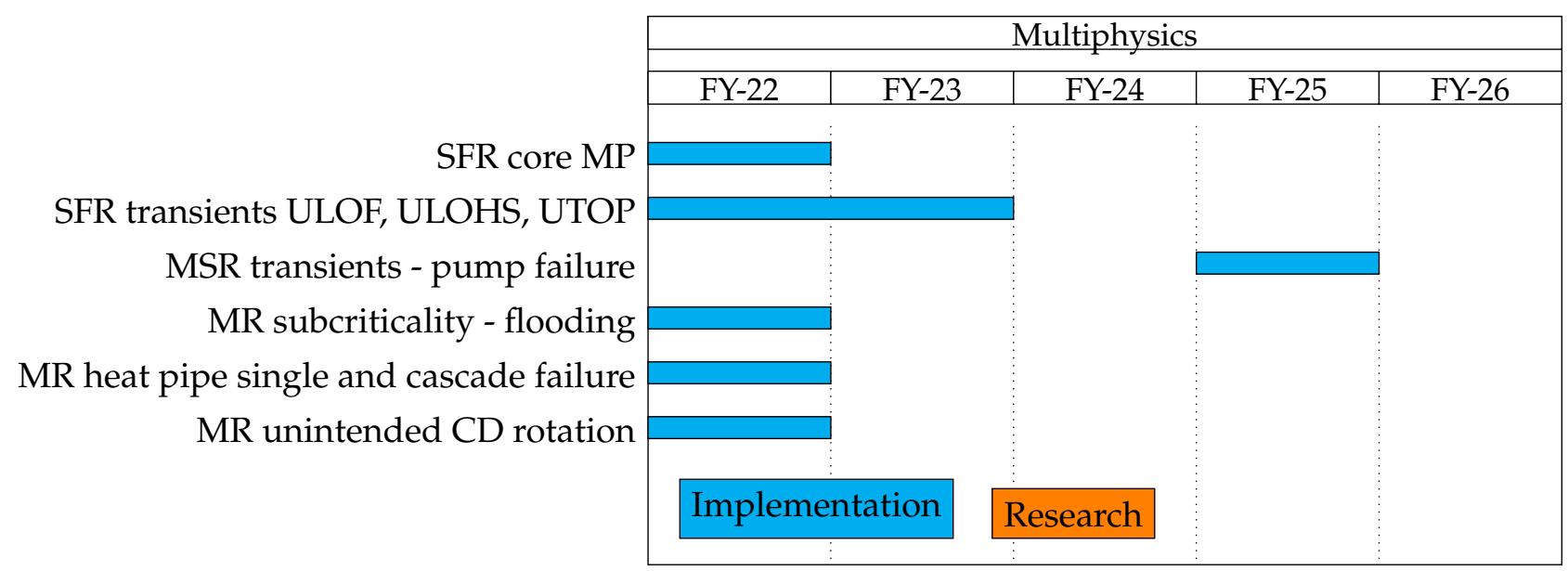

\section{VERIFICATION AND VALIDATION}

Verification - The following tests are included to verify integral capabilities and features as well as individual routines:

- Unit tests

- Integral system tests

- Analytic solution tests

- Method of Manufactured Solutions (MMS) [57]

- Code-to-code comparison - eigenvalue, reactivity worths, and power distribution are compared with the solutions from Monte Carlo codes.

- Numerical benchmarks

- Microreactors (EMPIRE [58], MegaPower [59], etc.)

- MSRs (MSRE [60], MSFR [61], etc.)

- HTRs (TREAT [62], VHTR [63], etc.) 
- SFRs (ABTR [64], Monju [65], etc.)

- PBR (HTR-10 [66])

- Performance tests

- ABTR [64] - sodium-cooled fast reactor problem with homogenized assemblies using 33 group cross sections

- C5 [67] - PWR-based thermal reactor problem with heterogeneous geometry using 11 or 23 group cross sections generated from Serpent2

- Empire [58] - thermal microreactor problem with heterogeneous geometry using 11 group cross sections

- MGEP [68] - MIT graphite exponential pile with heterogeneous geometry

- TREAT [62] - gas-cooled graphite-moderated high-temperature reactor with heterogeneous geometry based on the minimum critical core configuration

- Regression tests

Validation - The following tests are included to validate the code

- Validation benchmarks (available from ICSBEP, IRPhEP, etc.)

- SFR (ZPPR [69])

- MSR (MSRE [60])

- Microreactor (KRUSTY [70])

- HTR (HTTR [71], TREAT [62])

- PBR (HTR-10 [66])

- TREAT

\section{SOFTWARE QUALITY ASSURANCE}

The NQA-1 quality assurance standard involves the design, construction, and operation of nuclear reactors. Griffin is being developed to NQA-1 standards with the highest levels of rigor that would be applied to safety software with Quality Level 2. However, the Griffin owners will 
not be crediting the code as NQA-1 safety software and will not be issuing or supporting as safety software.

Griffin will use the same strategy as other MOOSE applications, as described in PLN-4005 [7]. This approach includes the automatic generation of the following documents:

- Software Test Plan (STP) and Software Test Description (STD)

- System Requirement Specification (SRS) - describes recommended approaches for the software requirements to explain the scope of the project, references made to other standards, definitions of specific terms used, background information, and essential parts of the software system.

- Requirement Traceability Matrix (RTM)

- System Design Description (SDD) - describes the necessary design information, including high-to-low level designs and specific methodologies for design and configuration management.

- Verification and Validation Report (VVR)

- Failure Analysis Report (FAR)

- User and theory manuals

We will follow good practices of software development: 1) develop and maintain the software at the INL HPC Gitlab repository with updates, error and bug fixes, etc.; 2) manage the repository for benchmark problems, documents, etc.; and 3) maintain a website for user error reporting and communication between users and developers.

Schedule - The SQA guidelines will be followed, keeping the items above updated throughout the entire development period. 


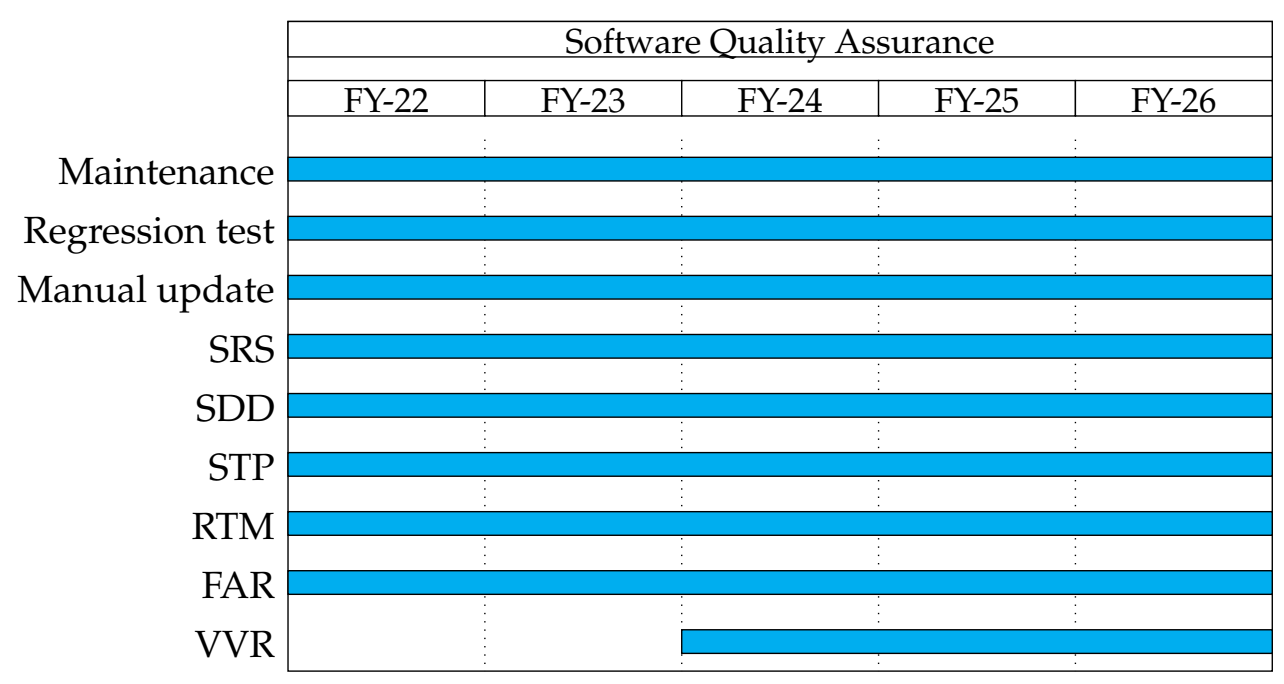

\section{REFERENCES}

[1] C.H. Lee and W.S. Yang, "MC²-3 : Multigroup Cross Section Generation Code for Fast Reactor Analysis," Nucl. Sci. Eng., vol. 187, pp. 268-290, June 2017.

[2] Y.S. Jung and C.H. Lee, "PROTEUS-MOC User Manual," Technical Report ANL/NE-18/10, Argonne National Laboratory, September 2018.

[3] A. Siegel et al., "Software design of sharp," in MEC 2007, (Monterey, CA), April 20072007.

[4] Y. Wang, S. Schunert, and V. Labouré, "Rattlesnake theory manual," Tech. Rep. INL/EXT-1742103, Idaho National Laboratory, 2018.

[5] F. N. Gleicher, J. Ortensi, et. al., "The coupling of the neutron transport application RATTLESNAKE to the fuels performance application bison," in International Conference on Reactor Physics (PHYSOR 2014), (Kyoto, Japan), May 2014.

[6] D. R. Gaston, C. J. Permann, J. W. Peterson, A. E. Slaughter, D. Andrs, Y. Wang, M. P. Short, D. M. Perez, M. R. Tonks, J. Ortensi, L. Zou, and R. C. Martineau, "Physics-based multiscale coupling for full core nuclear reactor simulation," Annals of Nuclear Energy, vol. 84, pp. 45-54, 2015.

[7] I. N. Laboratory, "Software Quality Assurance Plan - MOOSE and MOOSE-Based Applications," Procedure PLN-4005 Rev.8, Idaho National Laboratory, 52021. 
[8] ASME, “Quality Assurance Requirements for Nuclear Facility Applications," Standard 1a2009, ASME, 2009.

[9] C.H. Lee and Y.S. Jung, "Generation of the Cross Section Library for PROTEUS," Technical Report ANL/NE-18/2, Argonne National Laboratory, January 2018.

[10] J. Leppänen,, Development of a New Monte Carlo Reactor Physics Code. PhD thesis, Helsinki University of Technology, 2007.

[11] R. D. O'Dell, "Standard interface files and procedures for reactor physics codes. Version IV," tech. rep., Los Alamos National Laboratory, 91977.

[12] O. R. N. Laboratory, "SCALE: A modular code system for performing standardized computer analyses for licensing evaluation," Research Report ORNL/TM-2005/39, Version 5.1, Vols. IIII, Oak Ridge National Laboratory, TN, November 2006.

[13] J.L. Conlin, F.B. Brown and A.C. III Kahler and M.B. Lee and D.K. Parsons and M.C. White, "Updating the Format of ACE Data Tables," in LA-UR-12-22033, Los Alamos National Laboratory, June 2012.

[14] R. Morris, “Cubit 15.0 user documentation," tech. rep., ETI, UT, 2014.

[15] Y.S. Jung, C.H. Lee and M. A. Smith, "PROTEUS-NODAL User Manual," Technical Report ANL/NE-18/4, Argonne National Laboratory, September 2018.

[16] K. O. Ott and D. A. Meneley, "Accuracy of the Quasistatic Treatment of Spatial Reactor Kinetics," Nucl. Sci. Eng., vol. 36, pp. 402-411, 1069.

[17] O. Calvin, J. Ortensi, Y. Wang, S. G. S. Schunert, and M. DeHart, "Implementation of Depletion Architecture in the MAMMOTH Reactor Physics Application," Technical Report INL/EXT-19-55550, Idaho National Laboratory, 92019.

[18] B. J. Toppel, "The Fuel Cycle Analysis Capability REBUS-3," Technical Report Argonne-83-2, Argonne National Laboratory, March 1983. 
[19] S. Schunert, Y. Wang, J. Ortensi, V. Laboure, F. Gleicher, M. DeHart, and R. Martineau, “Control rod treatment for fem based radiation transport methods," Annals of Nuclear Energy, vol. 127, pp. $293-302,2019$.

[20] W. Oberkampf and C. Roy, Verification and Validation in Scientific Computing. Cambridge University Press, 012010.

[21] "Evaluation of the initial critical configuration of the HTR-10 pebble-bed reactor.," Tech. Rep. HTR10-GCR-RESR-001, International Reactor Physics Experiment Evaluation Project, 2006.

[22] E. Teuchert et al., "V.S.O.P ('94) Computer Code System for Reactor Physics and Fuel Cycle Simulation," Research Report Jülich-2897, Forschungszentrum Jülich, 1994.

[23] H. Gougar, W. Terry, A. Ougouag, W. Yoon, and R. Sen, "Pebbed6 - theory manual." Unpublished INL report, 2018.

[24] Y. Wang, J. Ortensi, S. Schunert, and V. Labouré, “A Pebble Tracking Transport Algorithm for Pebble Bed Reactor Analysis," in PHYSOR 2018: Reactor Physics paving the way towards more efficient systems, (Cancun, Mexico), April 2018.

[25] J. Ortensi, An Earthquake Transient Method for Pebble-Bed Reactors and a Fuel Temperature Model for TRISO Fueled Reactors. PhD thesis, Idaho State University, 2009.

[26] R. E. Alcouffe, F. W. Brinkley, D. R. Marr, and R. D. O’Dell, “User's Guide for TWODANT: A Code Package for Two-Dimensional, Diffusion-Accelerated, Neutral-Particle Transport," Technical Report LA-10049-M, Los Alamos National Laboratory, 1990.

[27] R. E. Alcouffe, "PARTISN calculations of 3D radiation transport benchmarks for simple geometries with void regions," Prog. Nucl. Eng., vol. 39 (2), pp. 181-190, 2001.

[28] R. J. Stamml'er et al., "HELIOS Methods," technical report, Studsvik Scandpower, 1998.

[29] P. K. Romano et al., “OpenMC: A State-of-the-Art Monte Carlo Code for Research and Development," Ann. Nucl. Energy, vol. 82, p. 90, 2015.

[30] R. MacFarlane and A. Kahler, "Methods for processing endf/b-vii with njoy," Nuclear Data Sheets, vol. 111, no. 12, pp. 2739 - 2890, 2010. Nuclear Reaction Data. 
[31] K. Yamaji, H. Koike, Y. Kamiyama, K. Kirimura, S. Kosaka, “Ultra-fine-group resonance treatment using equivalent dancoff-factor cell model in lattice physics code galaxy," J. Nucl. Sci. Technol., vol. 55, pp. 756-780, 2018.

[32] A. Yamamoto, "Evaluation of background cross section for heterogeneous and complicated geometry by the enhanced neutron current method," J. Nucl. Sci. Technol., vol. 45, pp. 1287$1292,2008$.

[33] M.L. Williams, "Resonance Self-shielding Methodologies in SCALE 6," Nucl. Technol., vol. 174, pp. 149-168, 2011.

[34] R. Sanchez and G.C. Pomraning, "A Statistical Analysis of the Double Heterogeneity Problem," Ann. Nucl. Energy, vol. 18 (7), pp. 371-395, 1991.

[35] H.S. Park, W.S. Yang and C.H. Lee, "Iterative Local Spatial Self-shielding Method of MC²-3 for Particulate Fuel Modeling," in MEC 2021, ANS, 2021.

[36] A. Hébert and G. Mathonniere, "Development of a third generation superhomogenization method for the homogenization of pressurized water reactor assembly," Nuc. Sci. Eng., vol. 2, p. $115,1993$.

[37] B. S. Kirk, J. W. Peterson, R. H. Stogner, and G. F. Carey, “libMesh: A C++ library for parallel adaptive mesh refinement/coarsening simulations," Engineering with Computers, vol. 22, no. 3-4, pp. 237-254, 2006. http://dx.doi.org/10.1007/s00366-006-0049-3.

[38] L. A. Schoof and V. R. Yarberry, “EXODUS II: A Finite Element Data Model, SAND92-2137," tech. rep., Sandia National Laboratory, December 1995.

[39] M.A. Smith and E.R. Shemon, "User Manual for the PROTEUS Mesh Tools," Technical Report ANL/NE-15/17 Rev 1.1, Argonne National Laboratory, 2016.

[40] H. Childs, E. Brugger, B. Whitlock, J. Meredith, S. Ahern, D. Pugmire, K. Biagas, M. Miller, C. Harrison, G. H. Weber, H. Krishnan, T. Fogal, A. Sanderson, C. Garth, E. W. Bethel, D. Camp, O. Rübel, M. Durant, J. M. Favre, and P. Navrátil, “Visit: An end-user tool for visualizing and analyzing very large data," in High Performance Visualization-Enabling Extreme-Scale Scientific Insight, pp. 357-372, Oct 2012. 
[41] J. Ahrens, B. Geveci, and C. Law, "Paraview: An end-user tool for large-data visualization," in The Visualization Handbook, 2005.

[42] The HDF Group, "Hierarchical data format version 5." http://www.hdfgroup.org/HDF5, 2000-2010.

[43] "PyGriffin in Workbench," Communication with N.E. Stauff , Argonne National Laboratory, 52021.

[44] B. T. Rearden, R. A. Lefebvre, A. B. Thompson, B. R. Langley and N.E. Stauff, "Introduction to the Nuclear Energy Advanced Modeling and Simulation Workbench," in MEC 2017, ANS, 2017.

[45] “Griffin Manual," Technical Report .

[46] K. S. Smith, "Assembly homogenization techniques for light water reactor analysis," Progress in Nuclear Energy, vol. 17,3, pp. 303-335, July 1986.

[47] M. Pusa and J. Leppänen, "Computing the Matrix Exponential in Burnup Calculations," Nuclear Science and Engineering, vol. 164, pp. 140-150, 2010.

[48] M. Pusa, "Rational Approximations to the Matrix Exponential in Burnup Calculations," Nuclear Science and Engineering, vol. 169, pp. 155-167, 2011.

[49] M. Pusa and J. Leppänen, "Solving Linear Systems with Sparse Gaussian Elimination in the Chebyshev Rational Approximation Method," Nuclear Science and Engineering, vol. 175, pp. 250-258, 2013.

[50] M. Pusa, "Higher-Order Chebyshev Rational Approximation Method and Application to Burnup Equations," Nuclear Science and Engineering, vol. 182, pp. 297-318, 2016.

[51] Y. Kawamoto, G. Chiba, M. Tsuji, and T. Narabayashi, "Numerical solution of matrix exponential in burn-up equation using mini-max polynomial approximation," Annals of Nuclear Energy, vol. 80, pp. 220-224, 2015. 
[52] T. M. Pandya, S. R. Johnson, T. M. Evans, G. G. Davidson, S. P. Hamilton, and A. T. Godfrey, “Implementation, Capabilities, and Benchmarking of Shift, a Massively Parallel Monte Carlo Radiation Transport Code," J. Comp. Phys., vol. 308, pp. 239-272, 2016.

[53] A. Novak et al., "Pronghorn theory manual," Tech. Rep. INL/EXT-18-44453, Idaho National Laboratory, 2018.

[54] R. Williamson, K. Gamble, D. Perez, S. Novascone, G. Pastore, R. Gardner, J. Hales, W. Liu, and A. Mai, "Validating the bison fuel performance code to integral lwr experiments," $N u$ clear Engineering and Design, vol. 301, pp. 232 - 244, 2016.

[55] J. E. Hansel, R. A. Berry, D. Andrs, and R. C. Martineau, “Sockeye: A 1-d heat pipe modeling tool," Tech. Rep. INL/EXT-19-55742-Rev000, Idaho National Laboratory, 92019.

[56] R. Hu, “A fully-implicit high-order system thermal-hydraulics model for advanced non-LWR safety analyses," Annals of Nuclear Energy, vol. 101, p. 174-181, 2017.

[57] P.J. Roache, "Code Verification by the Method of Manufactured Solutions," J. Fluids Engineering, vol. 124, pp. 4-10, 2002.

[58] M.D. DeHart, J. Ortensi and V. Labouré, "NEAMS Reactor Physics Assessment Problem," Technical Report INL/LTD-20-59184, Idaho National Laboratory, 2020.

[59] C.H. Lee and Y.S. Jung, "Neutronics Simulation of Micro Nuclear Reactors Using the HighFidelity Neutron Transport Code PROTEUS," Technical Report ANL-ABR-1 (ANL-AFCI173), Argonne National Laboratory, September 2006.

[60] P. Haubenreich and J. Engel and B. Prince and H. Claiborne, "MSRE Design and Operations Report, Part III, Nuclear Analysis," Technical Report ORNL-TM-0730, Oak Ridge National Laboratory, 1964.

[61] C. Fiorina et al., "Modeling and analysis of the MSFR transient behavior," Nucl. Energy, no. 64, p. $485-498,2014$.

[62] G.A. Freund, P. Elias, D.R. Macfarlane, J.D. Geier, and J.F. Boland, “Design summary report 
on the transient reactor test facility (TREAT).," Tech. Rep. ANL-6034, Argonne National Laboratory, 1960.

[63] T. K. Kim, W. S. Yang, T. A. Taiwo and H. S. Khalil, "Whole-Core Depletion Studies in Support of Fuel Specification for the Next Generation Nuclear Plant (NGNP) Core," Technical Report ANL-NE/RP-114598, Argonne National Laboratory, 2004.

[64] Y.I. Chang et al., "Advanced Burner Test Reactor Preconceptual Design Report,” Technical Report ANL-ABR-1 (ANL-AFCI-173), Argonne National Laboratory, September 2006.

[65] T. Hazama, A. Kitano, and Y. Kishimoto, "Criticality Evaluation for the Monju Restart Core," Nucl. Technol., vol. 179, p. 250-265, 2012.

[66] J. Ortensi et al., "Benchmark analysis of the htr-10 with the mammoth reactor physics application," Tech. Rep. INL/EXT-18-45453, Idaho National Laboratory, 2018.

[67] M.A. Smith and E.E. Lewis and B.C. Na, "Benchmark on Deterministic Transport Calculations without Spatial Homogenization: MOX Fuel Assembly 3-D Extension Case," Technical Report NEA/NSC/DOC(2005)16, OECD/NEA, 2005.

[68] S. Hauptman, Characterization of the MIT Graphite Exponential Pile. PhD thesis, Massachusetts Institute of Technology, 62019.

[69] H.F. McFarlane and S.B. Brumbach and S.G. Carpenter and P.J. Collins, "Benchmark Physics Tests in the Metallic-Fueled Assembly ZPPR-15," Nucl. Sci. Eng., no. 101, p. 137, 1989.

[70] J. Bass et al., “The 2020 Edition of the ICSBEP Handbook,” Technical Report INL/CON-1953744-Revision-0, Idaho National Laboratory, 2020.

[71] J. Ortensi, J. Cogliati, M. Pope, J. Bess, R. M. Ferrer, A. Bingham, and A. Ougouag, “Deterministic modeling of the high temperature test reactor," Research Report INL/EXT-10-18969, Idaho National Laboratory, 2010. 


\section{A. List of the Capabilities and Features Required for Griffin}

Table A1 : Summary of the Capabilities and Features of Griffin - Part 1.

\begin{tabular}{|c|c|c|c|}
\hline Class & Subclass & Capability/Feature & Section \\
\hline \multirow{3}{*}{$\begin{array}{l}\text { Functional } \\
\text { requirement }\end{array}$} & General req. & $\begin{array}{l}\text { MOOSE development standard } \\
\text { Linux platform } \\
\text { Maintained on INL HPC GitLab repository } \\
\text { C++ basis }\end{array}$ & 3.1 \\
\hline & Fast spec. req. & Cross section & \\
\hline & $\begin{array}{l}\text { Thermal spec. } \\
\text { req. }\end{array}$ & $\begin{array}{l}\text { Cross section } \\
\text { TRISO }\end{array}$ & \\
\hline \multirow{5}{*}{ Reactor Type } & SFR & $\begin{array}{l}\text { Core expansion } \\
\text { Control rod driveline expansion } \\
\text { Equilibrium cycle calculation } \\
\text { Reprocessing } \\
\text { Sodium void, control rod worth } \\
\text { Wrapped tube } \\
\text { Single or double ducts } \\
\text { Transients of interest (ULOF, ULOHS, UTOP) }\end{array}$ & 3.2 .1 \\
\hline & HTR/FHR & $\begin{array}{l}\text { Prismatic type } \\
\text { Neutron streaming via air holes (large CR hole) } \\
\text { Bypass flow } \\
\text { TRISO } \\
\text { Transients of interest? }\end{array}$ & 3.2 .3 \\
\hline & MSR & $\begin{array}{l}\text { Flowing fuel } \\
\text { Online fuel feed/removal } \\
\text { Transients of interest (pump failure,...) }\end{array}$ & 3.2 .2 \\
\hline & PBR & $\begin{array}{l}\text { TRISO } \\
\text { Online refueling } \\
\text { Pebble flow } \\
\text { Transients of interest? }\end{array}$ & 3.2 .4 \\
\hline & Microreactor & $\begin{array}{l}\text { Core expansion } \\
\text { Subcriticality for flooding condition } \\
\text { CD rotation control } \\
\text { Transients of interest (HP failure, CD rotation,...) }\end{array}$ & 3.2 .5 \\
\hline
\end{tabular}


Table A2 : Summary of the Capabilities and Features of Griffin - Part 2.

\begin{tabular}{|c|c|c|c|}
\hline \multirow{8}{*}{ Cross section } & General & $\begin{array}{l}\text { Up to }(n, 3 n) \\
\text { Fission, gamma produced energies } \\
\text { Broad group structures optimized for reactors }\end{array}$ & 4 \\
\hline & Fast & $\begin{array}{l}\text { Two-step process } \\
-M C^{2}-3 / T W O D A N T \\
\text { Replace TWODANT (R-Z) }\end{array}$ & 4.1 \\
\hline & Thermal & $\begin{array}{l}\text { CSAPI } \\
\quad \text { - Subgroup } \\
\text { - Resonance table } \\
\text { - Equivalence Dancoff-factor Cell (EDC) } \\
\text { TRISO }\end{array}$ & 4.2 \\
\hline & Equivalence & $\begin{array}{l}\text { SPH generation and parameterization } \\
\text { DF generation and parameterization } \\
\text { Form function }\end{array}$ & 4.3 \\
\hline & MC generated XS & Parameterization in temperature, $\mathrm{CR}$, burnup, ... & \\
\hline & Library & $\begin{array}{l}\text { Neutron } \\
\text { Gamma (including delayed gamma, beta) } \\
\text { Depletion } \\
\text { Nuclide data support } \\
\text { - ENDF/B-VII.0 } \\
\text { - ENDF/B-VII.1 } \\
\text { - ENDF/B-VIII.0 }\end{array}$ & 4.5 \\
\hline & Reconstruction support & Form function & \\
\hline & Format & ISOXML & 4.3 \\
\hline \multirow{3}{*}{ Geometry } & Regular & $\begin{array}{l}\text { Hierarchical structure with reporting ID } \\
\text { Mesh generated from the MOOSE mesh generator } \\
\text { Mesh displacement }\end{array}$ & 5 \\
\hline & Irregular & $\begin{array}{l}\text { Hierarchical structure with reporting ID } \\
\text { CUBIT mesh } \\
\text { Mesh displacement }\end{array}$ & 5 \\
\hline & PTT & $\begin{array}{l}\text { Tetahedral mesh support } \\
\text { material ID assignment at node level }\end{array}$ & 5 \\
\hline
\end{tabular}


Table A3 : Summary of the Capabilities and Features of Griffin - Part 3.

\begin{tabular}{|c|c|c|c|}
\hline \multirow{3}{*}{ Input \& output } & Input & $\begin{array}{l}\text { Mesh:exodus format } \\
\text { Cross section : ISOXML format } \\
\text { Automated reactivity calculation } \\
\text { Assign material to region } \\
\quad \text { - Material, cross section, depletion regions } \\
\text { CD rotation for microreactor }\end{array}$ & 6 \\
\hline & Output & $\begin{array}{l}\text { Convergence table (eigenvalue, flux, source) } \\
\text { Pin edit } \\
\text { Flux \& power distributions } \\
\text { Detector edit } \\
\text { Reconstruction }\end{array}$ & 6 \\
\hline & User interface & $\begin{array}{l}\text { Workbench/PyGriffin } \\
\text { Visualization } \\
\text { Automated error checks }\end{array}$ & 6 \\
\hline & Solver & $\begin{array}{l}\text { CFEM-SN, PN, diffusion } \\
\text { DFEM-SN } \\
\text { VNM (PN) } \\
\text { MOC 2D/3D } \\
\text { Diffusion-SPH } \\
\text { R-Z solver }\end{array}$ & 7.1 \\
\hline & $\begin{array}{l}\text { Boundary } \\
\text { condition }\end{array}$ & $\begin{array}{l}\text { Reflective, white, vacuum, albedo } \\
\text { Periodic } \\
\text { Time-dependent transport boundary source }\end{array}$ & 7.1 \\
\hline & $\begin{array}{l}\text { Angular } \\
\text { quadrature }\end{array}$ & $\begin{array}{l}\text { Gauss-Chebyshev } \\
\text { Level symmetric } \\
\text { Bickley-3 }\end{array}$ & 7.1 \\
\hline & Type & $\begin{array}{l}\text { Eigenvalue } \\
\text { Fixed source } \\
\text { Adjoint } \\
\text { Delayed neutron precursor drift } \\
\text { PBR low-resolution } \\
\text { PBR high-resolution }\end{array}$ & 7.1 \\
\hline & Particle & $\begin{array}{l}\text { Neutron } \\
\text { Gamma } \\
\text { Phonon }\end{array}$ & 7.1 \\
\hline $\begin{array}{l}\text { Calculation } \\
\text { capability }\end{array}$ & Acceleration & $\begin{array}{l}\text { NDA, DSA } \\
\quad \text { - Performance } \\
\quad \text { - Transient } \\
\text { Unstructured CMFD } \\
\text { Coarse-group (or multigroup) }\end{array}$ & 7.1 \\
\hline
\end{tabular}




\begin{tabular}{|c|c|c|}
\hline & $\begin{array}{l}\text { Preconditioning } \\
\text { Sweeper (DFEM) } \\
\text { Multilevel approach (transient w/ low-order solver) }\end{array}$ & \\
\hline Transient & $\begin{array}{l}\text { Full transient } \\
\text { IQS } \\
\text { Point kinetics }\end{array}$ & 7.1 \\
\hline $\begin{array}{l}\text { Performance } \\
\text { requirement }\end{array}$ & $\begin{array}{l}\text { Memory } \\
\text { Computation time } \\
\text { Max processor use: } 1,000 \text { processors } \\
\text { Max memory use: } 500 \mathrm{~GB}\end{array}$ & 7.1 \\
\hline Fuel cycle & $\begin{array}{l}\text { Depletion } \\
\text { - Chebyshev Rational Approx. Method (CRAM) } \\
\text { - Mini-Max Polynomial Approx. (MMPA) } \\
\text { - Predictor-corrector } \\
\text { - Pebble bed special depletion treatment } \\
\text { - Special depletion treatment for MSR } \\
\text { Shuffling \& batch system (non-equilibrium) } \\
\text { Equilibrium search } \\
\text { Inventory reporting }\end{array}$ & 7.2 \\
\hline $\begin{array}{l}\text { Perturbation } \\
\text { and sensitivity }\end{array}$ & $\begin{array}{l}\text { Sensitivity coefficient } \\
\text { First-order GPT }\end{array}$ & 7.3 \\
\hline Uncertainty quantification & & 7.3 \\
\hline
\end{tabular}


Table A4 : Summary of the Capabilities and Features of Griffin - Part 4.

\begin{tabular}{|c|c|c|c|}
\hline \multirow{3}{*}{ Multiphysics } & $\mathrm{T} / \mathrm{H}$ & SAM, Pronghorn & 8 \\
\hline & Structure & SAM, BISON & \\
\hline & Other & SAM, MOOSE Apps & \\
\hline Verification & & $\begin{array}{l}\text { Unit tests } \\
\text { Integral tests } \\
\text { Method of Manufactured Solutions (MMS) } \\
\text { Numerical benchmarks } \\
\text { - Microreactor (EMPIRE, MegaPower) } \\
\text { - MSR (MSRE, MSFR) } \\
\text { - HTR (TREAT, VHTR) } \\
\text { - SFR (ABTR) } \\
\text { - PBR (HTR-PM) } \\
\text { - C5 } \\
\text { Regression tests }\end{array}$ & 9 \\
\hline Validation & & $\begin{array}{l}\text { Analytic solution tests } \\
\text { Well-known benchmarks (ICSBEP, IRPhEP, etc.) } \\
\text {-SFR (ZPPR) } \\
\text {-MSR (MSRE) } \\
\text {-PBR (HTR-10) } \\
\text { Experimental or test reactors }\end{array}$ & 9 \\
\hline $\begin{array}{l}\text { NQA-1 } \\
\text { requirement }\end{array}$ & & $\begin{array}{l}\text { Software requirement specification (SRS) } \\
\text { Software design description (SDD) } \\
\text { Software test plan (STP) } \\
\text { Verification and Validation Report (VVR) } \\
\text { Requirement Traceability Matrix (RTM) } \\
\text { Failure Analysis Report (FAR) } \\
\text { User and method manuals } \\
\text {-Griffin } \\
\text {-Cross section library generation } \\
\text {-V\&V test reports }\end{array}$ & 10 \\
\hline Code licensing & & $\begin{array}{l}\text { Controlled and distributed by the Code } \\
\text { Oversight Group (COG) at INL and ANL }\end{array}$ & \\
\hline References & & Provide key references (documents) & \\
\hline
\end{tabular}

